\title{
Article \\ Meaningful Test \& Evaluation of Indoor Localization Systems in Semi-Controlled Environments
}

\author{
Jakob Schyga * (), Johannes Hinckeldeyn $\mathbb{D}^{\mathbb{D}}$, and Jochen Kreutzfeldt \\ Institute for Technical Logistics, Hamburg University of Technology, 21079 Hamburg, Germany; \\ jakob.schyga@tuhh.de, johannes.hinckeldeyn@tuhh.de, jochen.kreutzfeldt@tuhh.de \\ * Correspondence: jakob.schyga@tuhh.de
}

\begin{abstract}
Despite their enormous potential the use of Indoor Localization Systems (ILS) remains seldom. One reason is the lack of market transparency and stakeholders' trust in the systems' performance as a consequence of insufficient use of Test and Evaluation (T\&E) methodologies. The heterogeneous nature of ILS, their influences, and their applications pose various challenges for the design of a methodology that provides meaningful results. Methodologies for building-wide testing exist, but their use is mostly limited to associated indoor localization competitions. In this work, the TEE 4iLoc Framework is proposed - a methodology for T\&E of indoor localization systems in semi-controlled environments based on a system-level and black-box approach. In contrast to building-wide testing, T\&E in semi-controlled environments, such as test halls is characterized by lower costs, higher reproducibility, and better comparability of the results. The limitation of low transferability to real-world applications is addressed by an application-driven design approach. The empirical validation of the TEE 4iLoc Framework, based on the examination of a contour-based Light Detection and Ranging ILS, an Ultra Wideband ILS, and a camera-based ILS for the application of Automated Guided Vehicles in warehouse operation, demonstrates the benefits of T\&E with the TEE 4iLoc Framework.
\end{abstract}

Keywords: indoor localization; test and evaluation; methodology; benchmarking

\section{Introduction}

In many areas of daily life, we encounter Location Based Services (LBS), such as for navigation [1], delivery tracking [2], or contact tracing [3]. A localization system determines the location of an entity and therefore enables LBS. Indoor localization and Indoor Localization Systems (ILS) are referred to when localization is performed indoors. Mautz [4] presents several applications of indoor localization, such as the tracking of patients and equipment in hospitals, locating firefighters in a burning building, or optimizing material flow in warehouses.

Although ILS are attributed an enormous potential, their application in practice is rare. In contrast to outdoor applications, which are mostly based on the Global Positioning System (GPS), no technology has prevailed so far. Potortì et al. [5] point out the lack of standardized concepts, procedures, and metrics in test and evaluation of indoor localization systems as a reason for low market transparency, and consequently low trust of stakeholders, and a slow adoption rate. Likewise, Pasricha [6] shares this view and names the definition of application-domain-specific requirements as one of the key open research challenges in the field of indoor localization. Lymberopoulos et al. [7] point out that to promote the adoption of ILS, it is essential for Test and Evaluation (T\&E) to be meaningful.

What does it mean for test and evaluation to be meaningful, and what is the reason that localization systems are rarely subjected to meaningful test and evaluation? Meaningfulness in test and evaluation is characterized by the significance of the results to the stakeholders involved. To be meaningful to system developers, the test and evaluation results must be reproducible and comparable to other systems and experiments. From the perspective of an end-user or system integrator, test and evaluation serves to determine a system's 
suitability for a particular task. The main reason, that ILS are rarely subject to meaningful test and evaluation, is that test and evaluation of indoor localization systems is an extremely challenging task [8]. Mautz [4] terms the issue of finding the optimal localization system for an application as a multi-dimensional optimization problem. Figure 1 illustrates this problem, with the dimensions explained as follows.

- System dimension (Figure 1, left): The characteristics of a localization system depend on a variety of factors, such as the underlying technology (e.g. Bluetooth, vision), the measuring principle (e.g. time-of-flight, received signal strength), the positioning method (e.g. fingerprinting, pedestrian dead reckoning, multilateration), the implementation (hard- and software), and the system deployment (e.g. position and amount of anchor nodes), while data fusion techniques are commonly applied on different levels [9]. Deployment is of particular importance for infrastructure-based systems.

- Environment dimension (Figure 1, middle): The system's performance is affected by technical influences, such as occlusion, reflection, or radio interference which in turn are a consequence of the environment [10]. The environment for the application of ILS can vary widely, ranging from underground mines to multi-story office buildings or warehouses. The impact of the influencing factors on system performance depends on the system characteristics. Since the technology of indoor localization systems is also heterogeneous the dependencies on influencing factors also vary.

- Application dimension (Figure 1, right): To find a suitable system, the system performance must meet the requirements of the user. The type and level of requirements vary depending on the application and user. 16 potential user requirements, such as accuracy, latency, and integrity are described by Mautz [4].
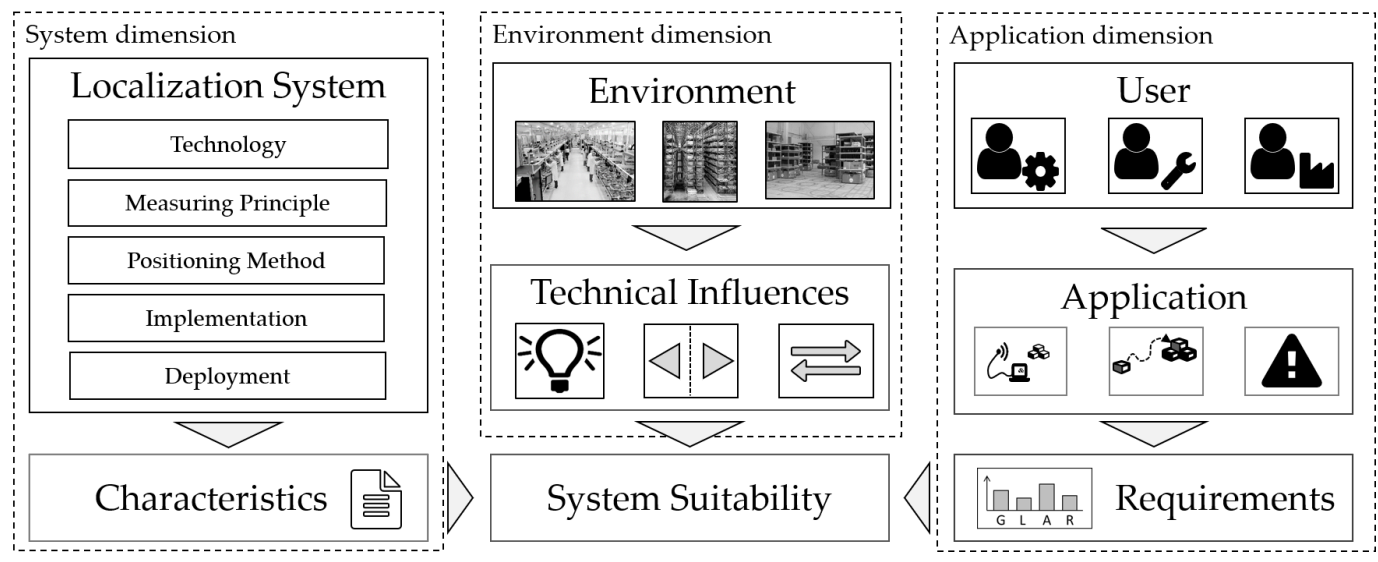

Figure 1. Matching requirements and localization systems - A multi-dimensional problem.

To achieve meaningful test and evaluation results the dimensions described must be taken into account. Significant spatial, financial, and organizational resources, as well as extensive metrology, statistical, data processing, and application domain expertise, are required for meaningful testing and evaluation [11]. Pasricha [6] points out that as a consequence, in practice mostly simple proof-of-concept tests are performed.

Methodologies are essential to support systematic test and evaluation and thus increase the meaningfulness of results. Methodologies for building-wide test and evaluation, such as the EvAAL Framework [12] and the EVARILOS Benchmarking Handbook [13] have been proposed. The ISO/IEC 18305 International Standard [14] was presented in 2016 to define a standardized procedure for test and evaluation of indoor localization systems with a focus on the end-user. Yet, publicly documented use of the named methodologies for test and evaluation is mostly limited to associated indoor localization competitions [7,15-17]. We published a work-in-progress paper on the topic of application-driven test and evaluation of indoor localization systems for warehouses for the Conference on Indoor Positioning and Indoor Navigation (IPIN) in 2021 [18]. This work expands the previously presented 
findings substantially by generalizing the methodology and by further elaborating all elements of the paper and the framework's components.

The main reason for the low adoption of methodologies are the high cost of buildingwide testing, especially for infrastructure-based ILS. For many localization systems and application environments, building-wide testing is required. Nonetheless, there are others, where the presence of rooms or other fixed structures is of minor importance or can be physically modelled. For such cases, tests are oftentimes performed in test halls that can be modified, for example, by the placement of certain objects [19-23]. In contrast to building-wide testing, this is considered a semi-controlled test environment, whereby semi-controlled means that some influences can be controlled, while others cannot. Since the environment can be controlled to achieve good performance results, the need for a methodology to achieve meaningful results is even more evident. However, a methodology for T\&E of ILS in semi-controlled environments does not yet exist.

In this work, the process of application-driven test and evaluation of ILS, including the involved stakeholders and their requirements are first analyzed in Section 2. Furthermore, test and evaluation in practice and existing methodologies are discussed. In Section 3 the TEE 4iLoc Framework is presented. The aim of the proposed methodology is to increase meaningfulness for T\&E in semi-controlled environments. It consists of several modules for specifying requirements, defining application-dependent scenarios, and for system-level, black-box T\&E of indoor localization systems. The empirical validation of the TEE 4iLoc Framework is described in Section 4, whereby a camera-based, a Light Detection and Ranging (LiDAR)-based, and a Ultra Wideband (UWB) ILS are examined, considering the application 'Automated Guided Vehicles in Warehouse Operation'. Finally, the proposed methodology is discussed and conclusions are drawn in Section 5.

\section{Test and Evaluation of Indoor Localization Systems}

Test and evaluation is in practice oftentimes carried out by system developers with the purpose of examining the performance of a localization system or its components [6]. Developers are particularly interested in the characteristics of the system itself and the differentiation and quantification of technical influencing factors on the system's performance. For users, it is paramount, how the system performs for a certain task in a given environment. To design a methodology that is meaningful, the stakeholders and their requirements regarding test and evaluation are first examined. Then it is presented how test and evaluation is performed in practice. Finally, the existing methodologies are discussed in terms of stakeholder requirements for test and evaluation.

\subsection{Stakeholders and Requirements}

Test and evaluation of localization systems can have a number of objectives, while the requirements depend on the stakeholder's perspective in the test and evaluation process [24]. The V-modell, commonly used as a management tool for software development and testing has been adapted to fit the application-driven test and evaluation for localization systems, as shown in Figure 2. The V-model enables the representation of the process with the associated stakeholders and their functions in different stages over time. Conclusions can be drawn from the evaluation back to the respective test stages. The stakeholders are divided into three categories - users, developers, and testers. 


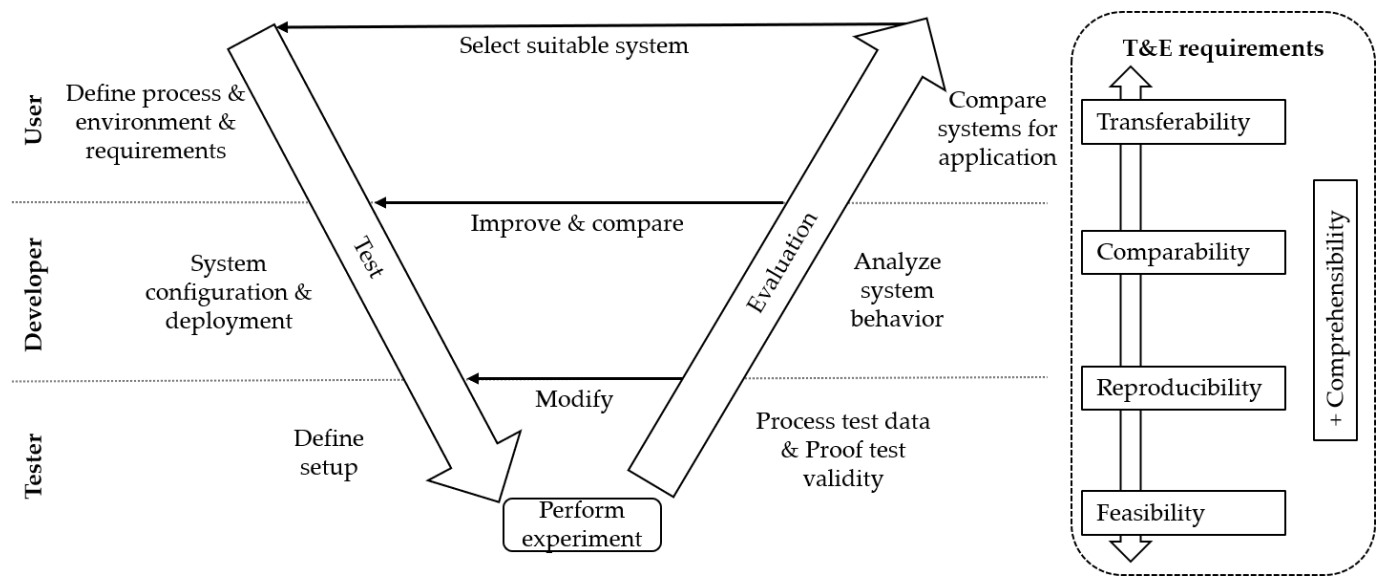

Figure 2. The V-Modell - Illustration of the application-driven T\&E process with the involved stakeholders, their functions, and requirements.

User

System users pursue a localization system to meet the requirements for a certain application. A user can either be an end-user or a system integrator. We consider an example from the logistics domain to clarify this division. An example of a system integrator could be the manufacturer of automated forklifts, while the localization system serves to enable the navigation of the forklift in warehouses. The system integrator integrates the localization systems into a product. An end-user, on the other hand, could be a warehouse operator who equips the warehouse with a localization system, to enable the tracking of goods and assets in warehouses. An end-user pursues localization systems either from the system developer or integrated into a higher-level product. Users strive to select the optimal system for their specific needs. They require that test and evaluation results are comparable to results of other systems, environments, or processes. We refer to this requirement as comparability. In addition, it is required that the results are transferable, i.e., that results are reproducible in real-world applications. To support transferability, users have to specify the considered application by defining the processes, environment, and system requirements.

Developer

Developers are soft- or hardware engineers who participate in the advancement of the technology. Sensor and algorithm development often results in prototype localization systems and components that must be tested and evaluated to draw conclusions about the performance. During application-driven test and evaluation, developers must specify the configuration and deployment of the localization system to meet the user's system requirements, ensuring that the results meet the requirements for comparability and transferability. Test and evaluation results are used by developers to evaluate and improve the system, its configuration, or its deployment. To assess system performance, developers require comparability of the test and evaluation result with other configurations, deployments, and environments. Comparability can only be achieved if test and evaluation results are repeatable and reproducible. Repeatability is the ability to achieve the same results when testing the same system in the same way in the same environment [14], while reproducibility is the ability to achieve the same results when testing the same system in a different environment [13]. In contrast to reproducibility, comparability allows comparison between the performance of systems based on the same or different scenarios. Non-repeatable testing can neither be reproducible. In the following, we will therefore refer to reproducibility, whereby repeatability is included. The reproducibility of tests depend not only on the test and evaluation process, but also on the system itself, and can only be achieved to a certain extent. 
Tester

Testers define a concrete test setup, perform experiments, processes data, and prove the validity of the results. They have to ensure the above-mentioned requirements for transferability, comparability, and reproducibility while at the same time enabling the feasibility of the test and evaluation. Feasibility measures the required spatial, financial, organizational, and human resources required to perform test and evaluation.

In addition, the comprehensibility of the processes and the data at each level of the V-Modell must be given for the respective stakeholders. Clearly defined processes, meaningful performance metrics, and documentation increase comprehensibility.

In practice, the stakeholder categories often overlap. In academic research, developers usually test their systems or system components themselves. Companies that are considered users or developers may also perform test and evaluation of the systems. The categories should be considered according to the role that a company, a department, or an individual plays during test and evaluation. The defined requirements, namely transferability, comparability, reproducibility, feasibility, and comprehensibility, are partially conflicting. Fully transferable test and evaluation requires T\&E to be performed in the operating warehouse based on the exact processes of the application. This would limit comparability, reproducibility, and feasibility. The defined requirements thus form a field of tension. Test and evaluation must be designed accordingly, while methodologies can be applied to support finding a suitable compromise.

\subsection{Test and Evaluation in Practice}

In the following, it is presented how test and evaluation of indoor localization systems is performed in practice. The limitations are pointed out and practical approaches to overcoming these limitations are discussed.

Localization systems and their components are commonly tested and evaluated by developers. The results are often published as experimental validation, e.g. in research papers. While suitable as a proof-of-concept, quantitative results must at least be regarded with caution [6,10]. Adler et al. [25] presented a myriad of testing procedures and evaluation metrics, based on 183 publications from the Indoor Positioning and Indoor Navigation (IPIN) conference of 2010 to 2014. The experiments range from simple office walks to realworld experiments in populated buildings. While absolute positioning accuracy is usually considered the main criterion, there are inconsistencies regarding the respective metric and the determination of the system's true position. Another survey was published by Bousdar Ahmed et al. [24] in 2020. In contrast to Adler et al. [25], Bousdar Ahmed et al. [24] analyzed the test and evaluation processes according to the characteristics defined in the ISO/IEC 18305 Standard [14]. While oftentimes not specified, the analyzed experiments vary widely regarding the building type, the number of floor levels, the type of motion, the number of evaluation points, the applied accuracy metrics, and the used ground truth. In addition, we analyzed the papers published at the IPIN conference in 2021 to examine the recent usage of methodologies. There were 85 full papers published at the IPIN 2021 conference. Empirical test and evaluation of the absolute localization accuracy was performed in 33 publications. None of the 33 publications is referring to an existing test and evaluation methodology. A table of the papers and their categorization into the underlying technology, ground truth, and test environment is provided in Appendix A. As a consequence of the diversity, comparability and reproducibility are low. Nonetheless, results are summarized in review papers [4,26-28] based on hardly comparable quantities [12]. T\&E results furthermore lack transferability, as concrete use-cases are rarely considered or specified. For system users, low transferability leads to the consideration or selection of systems and technologies that do not perform in practice as the test and evaluation results indicate [7]. To overcome these limitations, various approaches have been developed, each with its own advantages and disadvantages. The approaches are not mutually exclusive, but complementary. 


\section{Simulated data}

Test data can be generated by applying simulation models of the localization system and the environment. Evaluating localization systems and algorithms based on simulation data has the advantage of minimizing the costs of space, time, and equipment for the physical experiment setup while allowing full repeatability of experiments [29]. Nevertheless, simulations are always an abstraction of reality and are therefore object to certain limitations. For example, uncertainties in simulations are usually modelled by Gaussian noise [30]. In reality, measurement uncertainties result from complex interactions of various hardware and software components with the environment and go far beyond. While simulations are a useful tool for developers, they cannot replace experiments in the real world.

\section{Common data sets}

Inspired by the vast growth of robotics and artificial intelligence research, the indoor localization community has introduced benchmarking based on pre-recorded data sets [31]. Raw data from localization systems are provided, such as the Received Singal Strength (RSS) for Wi-Fi fingerprinting [32] or image data for visual localization [33]. The generation of raw data is separate from the actual localization algorithm. Hence, test and evaluation at the system level of a localization system cannot be performed. Benchmarking based on shared data sets has proven to be extremely useful to the research community. However, the benefit to the system user, who is mainly interested in how a system works as a whole, is limited.

\section{Indoor localization competitions}

Since the first IPIN conference in 2010, several competitions of indoor localization systems have been organized. In 2011, the EvAAL competition was held in conjunction with the IPIN conference, with the aim of evaluating indoor localization systems in the same environment with similar conditions [15]. Since then, the competition has been held annually, but was renamed to IPIN competition in 2014 [34]. The IPIN competition consists of several evaluation tracks focused on different use-cases and technologies. In 2016, for example, a track was designed to evaluate ILS for tracking of robots [35]. In 2017, the PDR Challenge in Warehouse Picking and in the following year, the xDR Challenge for Warehouse Operation were organized to evaluate systems for dead reckoning of pedestrians and vehicles in warehouse environments [36]. The PerfLoc competition was held between 2017 and 2018 by the U.S. National Institute of Standards and Technology (NIST), recording data from smartphone sensors $[16,17]$. The Microsoft Indoor Localization Competition was first held in 2014 in conjunction with the International Conference on Information Processing in Sensor Networks (IPSN) [7]. In 2021, the Microsoft Indoor Localization Competition attracted 1446 participants, competing based on data sets, which were collected by smartphone sensors in 212 different buildings [37]. A comprehensive overview of the characteristics of the aforementioned competitions is given by Ichikari et al. [36]. Competitions lead to good comparability of localization systems for the intended scenario and good transferability for the considered application domain or environment. On the other hand, transferability to other applications and comparability with other test and evaluation results remains limited. In addition, competitions based on the test and evaluation on a system-level are costly and hardly scalable [30].

It was shown, that quantitative performance results of test and evaluation are often not meaningful due to lack of transferability, comparability, and reproducibility. The approaches presented have been developed to mitigate these limitations, but rarely focus on the system user. Test and evaluation methodologies can be applied to systematize the $T \& E$ process and increase the meaningfulness of results. 


\subsection{Test and Evaluation Methodologies}

A methodology describes the general strategy for solving a problem, whereby decisions can be made within a set of rules [38]. A methodology incorporates methods and/or procedures and can be incorporated by approaches [38]. A benchmark is a well-defined procedure for conducting an experiment, collecting data, and computing metrics. In the following, the terms methodology and framework are used interchangeably.

The methodologies for test and evaluation of indoor localization systems are presented and discussed regarding the T\&E requirements. The systematic comparison is supported by considering the following characteristics.

- System-testing vs. component-testing: In contrast to component-testing, systemtesting describes how a localization system performs as a whole. There is no consideration of a localization system's individual components [14].

- Knowledge about the system's inner-workings: Designing test and evaluation processes considering the inner workings of a system leads to system-specific testing. In black-box testing, the test and evaluation process is designed without considering the inner workings of localization systems [14].

- Application-driven T\&E approach: Seltzer et al. [39] present three general approaches for application-driven T\&E. For the vector-based approach (1) a system vector, consisting of general performance metrics, is determined by microbenchmarks, while the application vector describes the requirements, which are determined by the application. The dot product of the vectors is considered a meaningful result. In the trace-based approach (2), the actual application influences the benchmark itself. Accordingly, the resulting system vector is application-specific. Finally, the hybrid approach (3) combines the trace-based and the vector-based approach.

- Test environment: In laboratory testing, ideally all relevant environmental influences for the test and evaluation can be controlled. In building-wide testing, a large space of the facility is used [14]. Semi-controlled environments allow partial control of environmental influences.

- Building specifications: In building-wide testing, the type of building has a significant impact on the system performance. Hence, the building specification needs to be considered.

- Provision of scenarios: Scenarios define conditions for an experiment.

- Entity to be Localized/Tracked (ELT): Requirements or considerations regarding the type of ELT.

- Ground truth specification: Requirements or considerations regarding the ground truth. The ground truth provides a location estimate that is considered to be the true location of an ELT. Commonly a reference system with significantly higher accuracy, or off-line surveyed evaluation points are applied as ground truth [14].

- Specification of path and test points: The system accuracy is usually determined by comparing the location estimate with the ground truth at test points along a certain path. The number, the position and the order of test points is relevant for the results $[6,7]$.

- Motion specification: Describe the way in which the ELT moves through the environment.

- Proposed metrics: Various metrics can be used to describe the performance of a localization system.

- Metrics applied for absolute position error: The absolute accuracy is usually considered the

describing the accuracy of a localization system.

- Consideration of Influences: Influences on the system performance can be considered for the experiment specification and/or system evaluation.

Table 1 provides a comparative overview of the test and evaluation methodologies by showing some background information, such as the related authors and the characteristics 
described above. In the following, we give a brief overview of the existing test and evaluation methods, their documented use, and their main advantages and disadvantages.

\section{EvAAL Framework}

The EvAAL Framework is a set of rules and metrics for the test and evaluation under complex and realistic conditions [12]. The core criteria of the EvAAL framework demand a natural movement of a human actor carrying the localization device, a realistic test environment, a realistic measurement resolution, and the third quantile of point euclidean error as a performance metric. Because the EvAAL framework is a fairly simple set of rules, its core criteria are often met in practical test and evaluation processes without explicit reference to the methodology. Its strengths consist in a high feasibility and comprehensibility. Only one metric is proposed and influences are not considered further, which limits comparability with other experiments and the transferability to real-world applications that differ from the test case. The EvAAL Framework is well suitable for use in competition. However, the results provide little insight into the system behavior and performance beyond the specific test case.

\section{EVARILOS Benchmarking Handbook}

The final version of the EVARILOS Benchmarking Handbook was published in 2015 as a result of the project EVARILOS $[10,13]$. The EVARILOS Benchmarking Handbook defines a scheme for specifying experiments and evaluating the experiments based on several metrics. The application domain is taken into account when specifying experiments and calculating the final performance score according to the application requirements. In addition to point accuracy, the EVARILOS Benchmarking Handbook also considers system latency, energy efficiency, and robustness to changes in radio interference and environment. The EVARILOS Benchmarking Handbook is designed thoroughly, taking into account various metrics and influences, leading to good comparability and reproducibility. Application-specific definition of scenarios and score calculation is enabled. Although the EVARILOS Benchmarking Handbook is sophisticated and several tools are provided [11,40], the methodology has only been applied in the EVARILOS project itself, the EVARILOS Open Challenge [41], the EvAAL competition, and the Microsoft Indoor Localization Competition in 2014 [42].

\section{ISO/IEC 18305:2016 International Standard}

Building on the findings of EVARILOS, the ISO/IEC 18305:2016 International Standard was published in 2016 to define terms, scenarios, metrics, and reporting requirements, and to provide considerations for test and evaluation of generic localization systems [14]. The ISO/IEC 18305 is focused on a system-level, black-box, and building-wide test and evaluation approach. Buildings are categorized in five types, such as underground mines and warehouses and ELT are categorized in 'Object', 'Person', and 'Robot'. Different mobility modes for the ELT-type person are defined, such as backward walking and sidestepping. The building types, ELT, and mobility modes are combined to 14 test scenarios, without further consideration of the robot class. The standard has been discussed in several publications $[5,24,43]$. However, the publicly documented use of the ISO/IEC 18305 is limited to the PerfLoc competition [16]. Test and evaluation according to the ISO/IEC 18305 leads to meaningful results for both, the system developer and the user

Many efforts have been made to address the problem of meaningful test and evaluation of indoor localization systems. The approaches range from simple proofs-of-concept to extensive experiments in real-world environments in conjunction with competitions based on open data sets. Methodologies aim to systematize test and evaluation, thereby increasing the benefits for the stakeholders. For all methodologies presented, the high costs and effort for building-wide testing are a major limiting factor. 
Table 1. Comparisons of methodologies for test and evaluation of indoor localization systems.

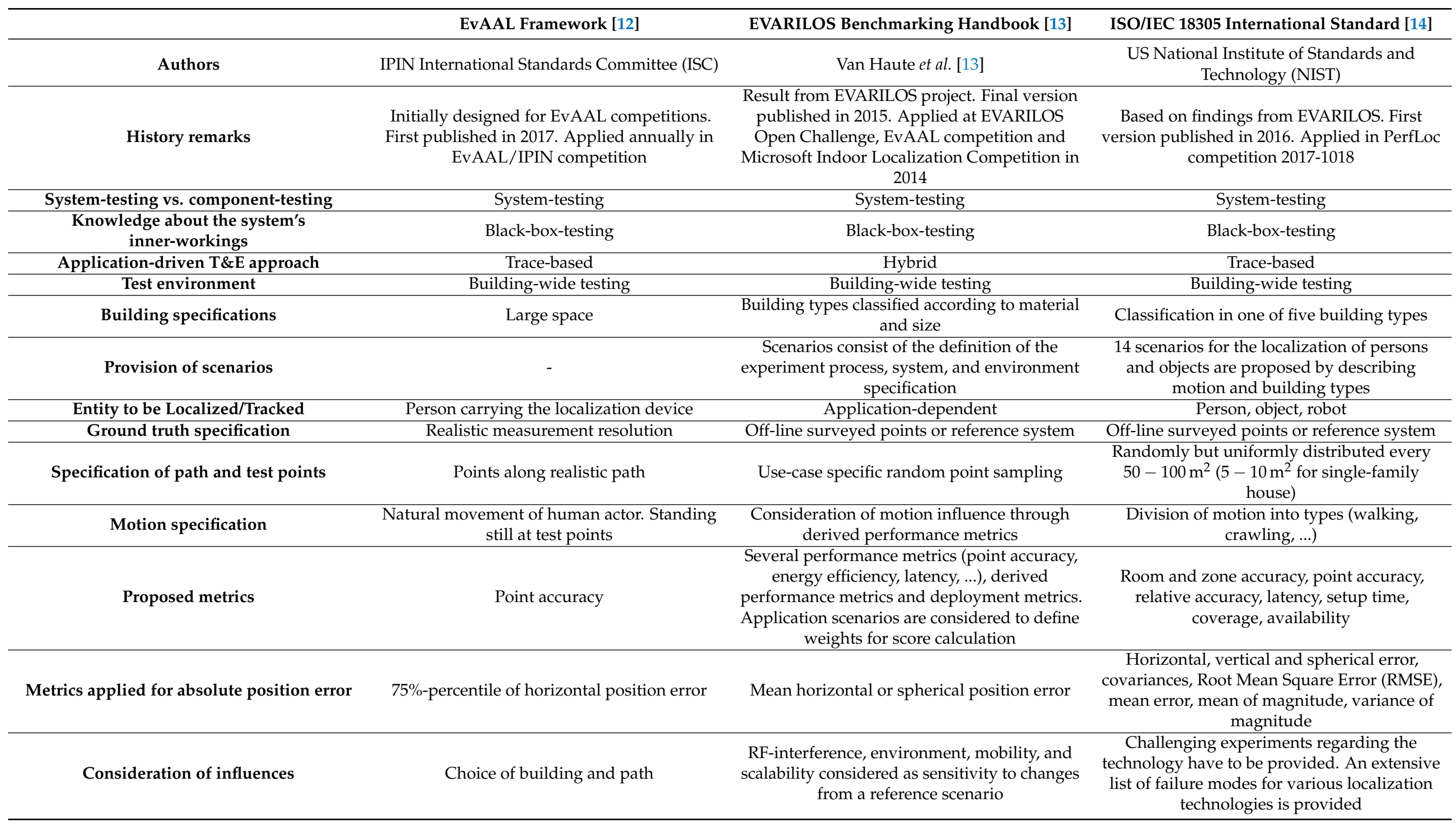




\section{Test and Evaluation with the TEE 4iLoc Framework}

In the following, the TEE 4iLoc Framework is presented. Unlike existing methodologies for black-box, system-level, the TEE 4iLoc Framework is not designed for building-wide testing. Especially for RF-based systems the building type, and structures are of great importance to the system performance. However, building-wide testing has the disadvantages of low reproducibility, comparability, and feasibility. In practice, test and evaluation of localization systems is often performed in test halls [19-23]. In this work, the term semi-controlled test environment is introduced as a category for test environments for T\&E of indoor localization systems. Semi-controlled test environments are indoor facilities, such as test halls, large rooms, or large open spaces in a building, which provide an open test area with a modifiable environment. There are usually various constraints on the control of the environment, such as the facility itself, the available objects, or the deployment of system infrastructure. It is assumed, that in semi-controlled test environments $T \& E$ can be performed with some degree of similarity in terms of factors influencing the system performance. A major challenge for T\&E is such environments is to produce results that are meaningful to both system developers and users. The following guidelines were established to incorporate the results from the previous analysis of test and evaluation (Section 2) into the TEE 4iLoc Framework.

1. Application focus: Test and evaluation that is meaningful to a system user can only be performed with respect to an application. The heterogeneity of indoor localization applications must be considered.

2. Technology openness: A system user is not interested in the inner mechanisms of a localization system as long as it works according to the system requirements. Consequently, an application-driven methodology must be open to different localization system technologies.

3. Stakeholder focus: System developers and testers require different information from a localization system than system users. A methodology should consider the needs of all stakeholders involved, while a stakeholder should only receive relevant information.

4. Modular design: To find broad acceptance and thus increase comparability, a methodology must be practicable for system users and developers from academic research and industry. Modularity supports feasibility for different stakeholders and use cases while ensuring comparability through clearly defined processes and interfaces.

First, considerations of the TEE 4iLoc Framework are stated with respect to the previously discussed characteristics of test and evaluation. Then, the framework architecture and components are explained. An overview of the terminology can be found in Appendix B.

\subsection{Characteristics}

The characteristics of the TEE 4iLoc Framework are discussed to provide a comprehensible overview and enable the comparison to the previously presented methodologies in Table 1.

System-testing vs. component-testing and knowledge about the system's inner-workings

The performance of the components of a localization system or the systems innerworking are not relevant from a system user's point of view as long as the system meets the requirements. Hence, a system-level and black-box approach is used. The output of the localization system is in the form of absolute position and/or heading data with timestamp.

Application-driven T\&E approach

The TEE 4iLoc Framework is based on a hybrid approach according to Seltzer et al. [39] with the goal of determining the suitability of a localization system for an application. Consequently, it influences both testing and evaluation. 
Test environment and building specifications

As previously mentioned, the TEE 4iLoc Framework is designed for T\&E in semicontrolled environments. It is required that a rectangular test area is provided in an open space. A test area of at least $50 \mathrm{~m}^{2}$ is suggested to enable the guidance of the ELT, object placement, and evaluation of location-dependent errors.

\section{Provision of scenarios}

The provision of predefined test scenarios increases reproducibility and comparability. However, the transfer to a different practical applications is limited. Hence, functions are provided to allow flexible but systematic definition of application-dependent test scenarios. In addition, a reference scenarios is provided.

\section{ELT and motion specification}

ELT and its motion can be specified flexibly depending on the application. The classes 'Person', 'Object', and 'Robot' for entities are adopted as reference classes from the ISO/IEC 18305 [14]. In addition, 'Vehicles', such as manually guided industrial trucks are considered as an entity class.

\section{Ground truth specification}

The TEE 4iLoc Framework requires a reference localization system. For off-line surveyed points, no timestamp is provided automatically for ground truth data, which is of particular importance for dynamic measurements. In addition, a reference system provides continuous localization data, which is relevant for system developers and testers to analyze system behavior and to prove test validity. As specified in the ISO/IEC 18305 [14], the ground truth accuracy should be at least one order of magnitude higher than that of the localization system.

Specification of path and test points

The heading of a localization system can have a significant influence on the localization accuracy. Hence, poses $(x, y, y a w)$ are considered instead of points $(x, y)$. A path is defined by evaluation poses, which are traversed by the ELT one after the other. The suggested number of evaluation poses is one per square meter. This number is not fixed and should be adjusted to reach statistically significant results for the localization system, test environment, and the test and evaluation procedure. Guidelines for the selection of evaluation points are provided by de Poorter et al. [44]. The poses can be selected based on various criteria, such as random grid-based sampling or by defining an application-dependent path.

Proposed metrics and metrics applied for absolute position error

The localization accuracy is considered the most important performance characteristic of a localization system. Unlike the EVARILOS Benchmarking Handbook [13] and the ISO/IEC 18305 [14], the TEE 4iLoc Framework focuses only on localization accuracy. Metrics other than localization accuracy are not precluded. However, for these, reference is made to the considerations provided in the EVARILOS Benchmarking Handbook [13] and ISO/IEC 18305 [14]. The TEE 4iLoc Framework considers horizontal position $(x$ and $y)$, vertical position $(z)$, and heading (yaw), resulting in four Degrees-of-Freedom (4-DoF). A comprehensive list of the accuracy metrics considered can be found in Section 3.8.

\section{Consideration of Influences}

Influences are taken into account by defining scenarios. Analysis of influences on system performance is enabled by comparing performance metrics from different scenarios, e.g., by calculating sensitivity values as suggested in the EVARILOS benchmarking Handbook [11], or by multifactor analysis [45]. In this work, the analysis of influences is not addressed in depth. 


\subsection{Architecture}

The TEE 4iLoc Framework is designed in a modular way, consisting of seven function modules $((\mathbf{a})-(\mathbf{g}))$. Each module consists of several functions that result in output data that is further processed by subsequent modules. The framework architecture is shown in Figure 3.

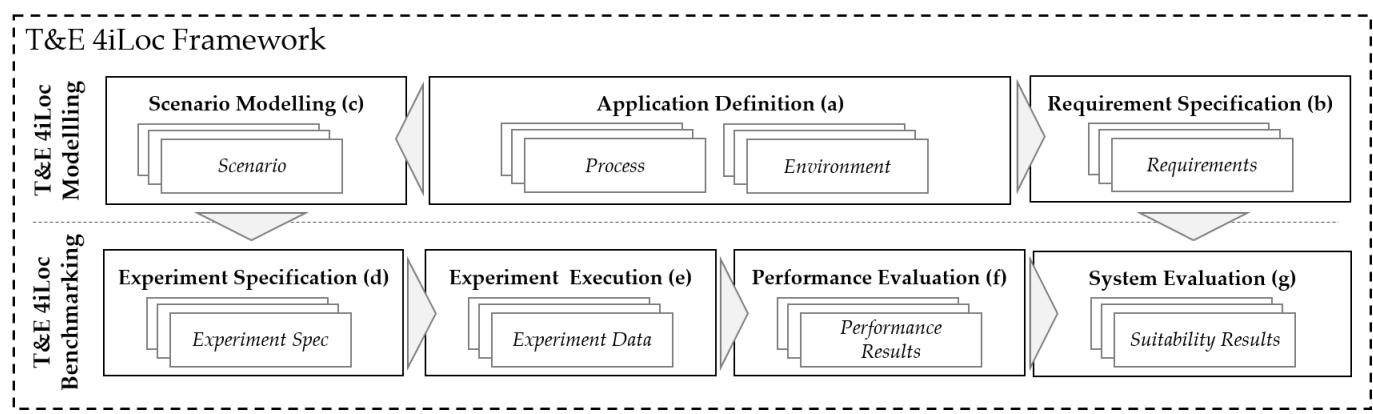

Figure 3. Architecture of the TEE 4iLoc Framework.

The modules are grouped into two procedures. The TEE 4iLoc Modelling Procedure contains the Application Definition, Scenario Definition, and the Requirement Specification. The TEE 4iLoc Benchmarking Procedure contains the Experiment Specification, Experiment Execution, Performance Evaluation, and System Evaluation. The division into the two procedures enables the distinction between application-dependent T\&E by using both, and application-independent T\&E, by using only the TEE 4iLoc Benchmarking Procedure.

Application-dependent T\&E is enabled by the TEE 4iLoc Modelling Procedure. First, the application is defined by describing the Processes and the Environment as part of the Application Definition. Application-dependent evaluation is achieved by defining system Requirements, as a result of the Requirement Specification. On the other hand testing is influenced by defining a Scenario as part of the Scenario Definition. The TEE 4iLoc Modelling Procedure leads to a testbed-independent abstraction of an application for the test and evaluation.

The TEE 4iLoc Benchmarking Procedure can be based on a Scenario and Requirements for application-dependent $T \& E$, or on freely defined scenarios for application-independent T\&E. The testbed-independent scenario is transferred to a testbed-dependent Experiment Spec. Then, the experiment is performed based on the functions of the Experiment Execution, resulting in a set of Experiment Data consisting of continuous, timestamped localization and reference data. The calculation of performance metrics and the data visualization is done in the Performance Evaluation. The Performance Evaluation is focused on developers and tester and provides a holistic view of the system behavior. Finally, the System Evaluation matches Requirements and Performance Results by providing and comparing the performance metrics relevant to an application.

In the following, the briefly introduced modules (a)-(g) of the TEE 4iLoc Framework are explained in more detail by defining their functions (1)-(3) and output data.

\subsection{Application Definition (a)}

It is critical whether test and evaluation is performed for tracking customers in a supermarket, firefighters in a burning building, or for navigating an autonomous forklift. The goal of the Application Definition is to describe exactly what the Application or Application domain Under Consideration (AUC) looks like, whereby an application domain contains multiple applications. The functions of the Application Definition are shown in Figure 4. 


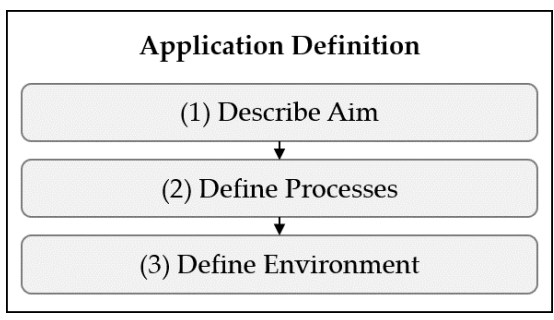

Figure 4. Functions of the module Application Definition (a).

First, the aim of the AUC is described (1). Then, the application is broken down, by defining the respective Processes (2) and the Environment (3). Depending on the aim of the test and evaluation procedure, it may be reasonable to further divide processes and environments.

To illustrate the functions of the module and their relevance, the example of customer tracking in a supermarket is considered. The aim of the AUC could be to gain transparency of customer behavior by tracking their movements during the shopping process. Exemplary Processes are 'searching for products', 'picking products', or 'comparing prices'. The Environment is defined by describing relevant dynamics and objects in a supermarket. If relevant, the supermarket environment could be further divided, e.g. into shopping and checkout areas.

\subsection{Requirement Specification (b)}

To determine the suitability of a system for AUC, specific performance requirements must be derived. As discussed in section 3.1, the TEE 4iLoc Framework focuses on performance requirements related to localization accuracy. Hence, the absolute accuracy of the following parameters is considered:

- Horizontal position $(x, y)$;

- $\quad$ Vertical position $(z)$;

- $\quad$ Spherical position $(x, y, z)$;

- Heading (yaw).

Three functions are defined to specify the requirements (Figure 5b). First, localization functions must be derived (1). Second, relevant requirement parameters are selected (2) by checking whether the accuracy of a parameter is relevant for realizing the localization function. Requirements can be marked as 'must' or 'shall'. Then, their magnitudes are defined with the respective confidence level (3). The magnitudes depend on the exact localization functions. Thus generic rules can hardly be defined. In general, the dimensions of the ELT and the size of the region of interest are relevant. The confidence $p$ is described by multiple of the standard deviation for a normal distributed measure. To ensure reliable processes, the Six Sigma ( $6 \sigma$ ) methodology has become established as a quality management tool in the industry [46]. The goal of Six Sigma is to improve the quality of processes to such an extent that only 0,00034\% of processes are defective. For the TEE 4iLoc Framework, the description by sigma values is adopted to define the following confidence levels for localization data:

1. Very low $(2 \sigma): p<93,3 \%$;

2. Low $(3 \sigma): p>93,3 \%$;

3. Moderate $(4 \sigma): p>99,38 \%$;

4. High $(5 \sigma): p>99,977 \%$;

5. Very high $(6 \sigma): p>99,9997 \%$.

It is important to note that the confidence level for each individual position estimate of a system is not equal to the confidence in a localization function, since incorrect location estimates can be compensated at a higher level of the application. While the requirements for tracking people or objects to gain transparency about processes are rather loose, the requirements for controlling robots or vehicles are rather strict. 
Considering the example of customer tracking in a supermarket, a localization function could be to determine the horizontal position of customers in the supermarket over time. The relevant requirement parameter is consequently the person's horizontal position, which should not exceed the shelf width, from which the magnitude of the accuracy requirement can be derived.

An application or process can usually be implemented in different ways. It is therefore not possible to prove whether a set of requirements is correct or not, until the AUC has been implemented. However, by defining the processes and localization functions, the determination of the performance requirements is comprehensible. Requirements can be combined to define the requirements of a process, with several localization functions, an application with several processes, or an application domain consisting of multiple applications.

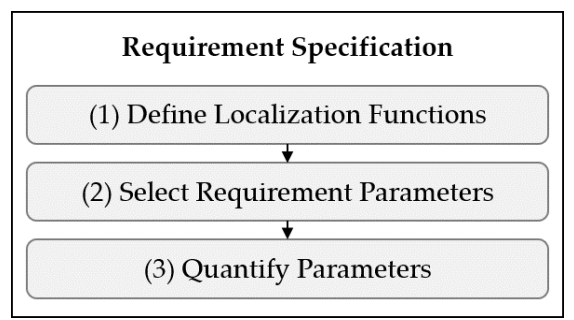

(b)

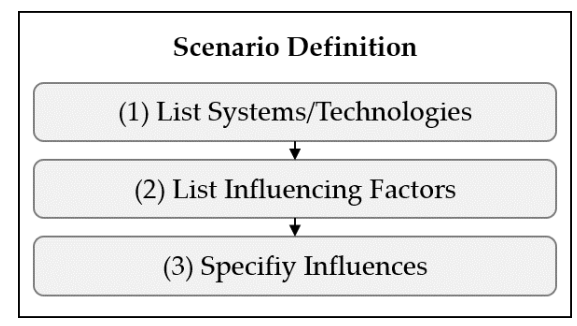

(c)

Figure 5. Functions of the module Requirement Specification (b) and Scenario Definition (c).

\subsection{Scenario Definition (c)}

A Scenario in the TEE 4iLoc Framework is an abstraction of an AUC. The aim of a Scenario is to enable application-dependent testing with the TEE 4iLoc Benchmarking Procedure. It contains a description of the application-dependent influences on the performance of localization systems.

The Scenario Definition is based on three functions shown in Figure 5c. First, systems that are considered to be tested (System Under Test - SUT) are listed (1). Then, the application-driven influencing factors are listed (2) and specified (3) by matching environmental and process influences with the AUC to define an application-dependent scenario. In contrast to technical influencing factors, such as occlusion or reflection, applicationdriven influencing factors are derived from the applications' processes and environment have a practical character.

A static, multi-camera system, as presented by Sun et al. [47] and a UWB localization system are considered for the application of tracking customers in a supermarket. Application-driven influencing factors on the system's localization accuracy, related to the process, could be the type of entity to be localized, the type of motion of the entity, and the type of path it follows. In terms of the environment, static objects, dynamic objects, and lighting conditions may be considered application-dependent influences on the system's performance. Finally, to define an application-dependent scenario, the application-driven influencing factors are specified with respect to the AUC. For the given example, the ELT class to be selected is 'Person', while the motion type is described by walking at moderate speed. Table 2 gives an example overview of an application-driven scenario for tracking customers in a supermarket. 
Table 2. Exemplary application-dependent scenario for the application of customer tracking in a supermarket. Camera and UWB ILS are considered as localization systems.

\begin{tabular}{c|ccc|cc}
\hline & \multicolumn{3}{|c}{ Process Influences } & \multicolumn{2}{c}{ Environment Influences } \\
\hline Factors & ELT & Motion & Path & $\begin{array}{c}\text { Static } \\
\text { Objects }\end{array}$ & $\begin{array}{c}\text { Lighting } \\
\text { Condition }\end{array}$ \\
\hline Specification & Person & Walking & $\begin{array}{c}\text { Straight and } \\
\text { curves on } \\
\text { horizontal } \\
\text { plane }\end{array}$ & Shelves & $\begin{array}{c}\text { Daylight and } \\
\text { artificial light }\end{array}$ \\
\hline
\end{tabular}

Scenarios enable an application-dependent specification of experiments and can be reused in test and evaluation procedures in different facilities. By specifying experiments based on systematically defined Scenarios, reproducibility and comparability are supported, while application-driven influencing factors are considered to increase the transferability of the results.

A Reference Scenario is provided to establish a standard case for test and evaluation procedures. The reference scenario aims for minimal application-driven influences on generic localization systems. The Reference Scenario is provided in Table 3. There are neither static nor dynamic objects to be placed. As for the the process influences, the Reference Scenario requires a robot, which is slowly following a path. The path is defined by randomly distributed evaluation poses. The ELT class 'robot' is chosen because the automatic execution leads to an optimal path accuracy and thus increases repeatability of an experiment. The ELT has to be static inside the tolerance area of the evaluation pose. The Reference Scenario can be extended depending on the influencing factors on the system performance of a SUT.

Table 3. Reference Scenario for the TEE 4iLoc Framework.

\begin{tabular}{c|ccc|cc}
\hline & \multicolumn{3}{c}{ Process Influences } & Environmental Influences \\
\hline \multirow{2}{*}{ Factors } & ELT & Motion & Path & $\begin{array}{c}\text { Static } \\
\text { Objects }\end{array}$ & $\begin{array}{c}\text { Dynamic } \\
\text { Objects }\end{array}$ \\
\hline \multirow{2}{*}{ Specification } & Robot & $\begin{array}{c}\text { Low velocity, } \\
\text { atatic mea- } \\
\text { surement }\end{array}$ & $\begin{array}{c}\text { Randomly } \\
\text { distributed } \\
\text { evaluation } \\
\text { poses }\end{array}$ & None & None \\
\hline
\end{tabular}

The TEE 4iLoc Modelling is completed by carrying out the Application Definition, Requirement Specification, and Scenario Modelling. Application-dependent Scenarios and Requirements can be used for application-dependent test and evaluation with the TEE 4iLoc Benchmarking Procedure. The TEE 4iLoc Benchmarking Procedure is elaborated in the following.

\subsection{Experiment Specification (d)}

A Scenario is transferred into a Experiment Spec by the Experiment Specification. Unlike a Scenario, a Experiment Spec is dependent on the testbed. The functions of the Experiment Specification are shown in Figure 6d. 


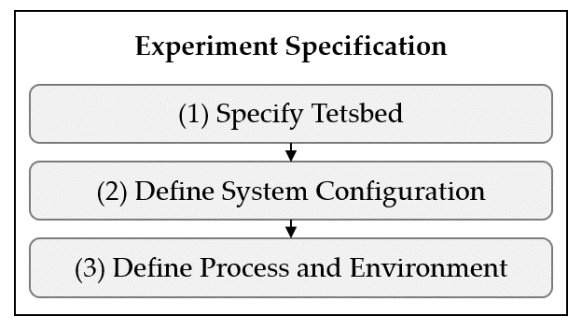

(d)

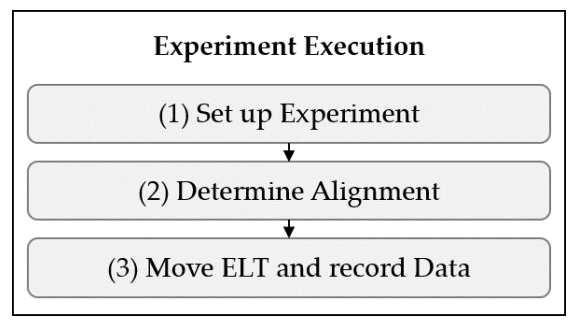

(e)

Figure 6. Functions of the module Experiment Specification (d) and Experiment Execution (e).

First, the testbed is specified (1) by defining the dimensions of the facility, the rectangular test area, available ELT, the environmental control parameters, and the reference system. The provisions of photos and layouts of the test facility are suggested. Next, the configuration of the SUT need to be defined (2) by describing the position of hardware components and the configuration of software parameters. To achieve a realistic setup, it is essential to define the system configuration according to the instructions from the system developer, taking into account the AUC. Finally, the benchmarking process and the environment configuration are defined (3) considering the Scenario and testbed specification. The environment is defined by describing the implementation of the environment influencing factors, such as the type and position of static objects. The benchmarking process is defined accordingly, e.g. by specifying the selected ELT, its motion, and path through the calculation of evaluation poses. Tolerance values must be defined as the minimal closeness for reaching an evaluation pose.

It is suggested to sample the evaluation poses on a grid with the grid size $g$, which defines the distance between the gridlines. The horizontal position tolerance $b$ should be less than half of the grid size to avoid overlapping of tolerance areas. The heading of the evaluation poses should be chosen between a fixed set of options, such as multiple of $90^{\circ}$. This allows for systematic analysis of the location-dependent localization accuracy. For random grid-based sampling, the evaluation poses are randomly selected from the grid points and the options for the heading. Figure 7 illustrates the exemplary result of random grid-based sampling of the evaluation poses on a rectangular test area for six evaluation poses. Depending on the path type defined in the Scenario, different sampling methods can be applied. An overview of various sampling methods is provided in the EVARILOS Benchmarking Handbook [13].

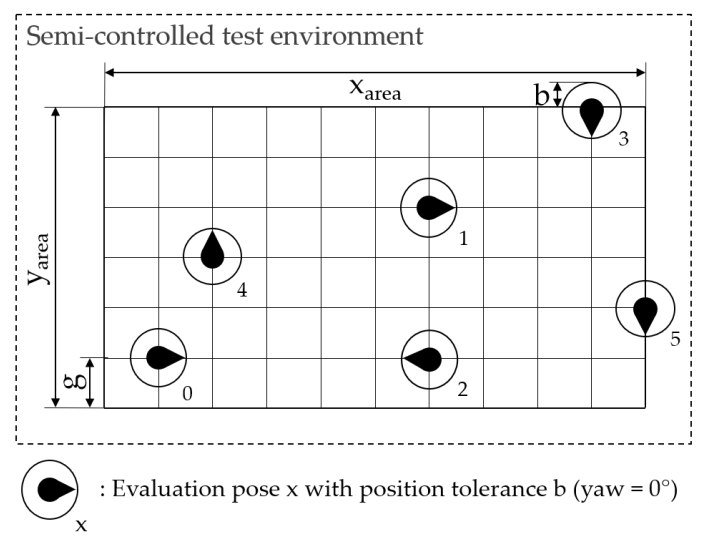

Figure 7. Random grid-based sampled evaluation poses. The arrow points into the heading directions of an evaluation pose.

The specified testbed, system configuration, environment configuration and the benchmarking process fully specify an experiment. An experiment can be repeated in the same facility by following the information of an Experiment Spec. 


\subsection{Experiment Execution (e)}

The Experiment Execution serves to create Experiment Data based on the Experiment Spec. The Experiment Execution consists of three functions as shown in 6e. First, the experiment is set up (1) according to the Experiment Spec by:

- Setting up the test environment according to the environment specification;

- Deploying and configuring the localization systems according to the system specification;

- Calibrating the reference system;

- Synchronising the clocks of the reference system and localization system;

- Setting up the ELT to allow its localization with the reference and localization system.

Next, the alignment between the global origins of the coordinate systems of the localization system $O_{l o c}$ and the reference system $O_{r e f}$ musst be determined (2). This is done by recording localization and reference data at a number of uniformly distributed points on the test area. It is proposed, to compute the transformation matrix $T_{r e f, l o c}$, by applying the Umeyama-Alignment [48] without scaling. If the mean pose errors would be eliminated as a bias of the Experiment Data, systematic location-dependent errors would be neglected. The alignment is illustrated in Figure 8.

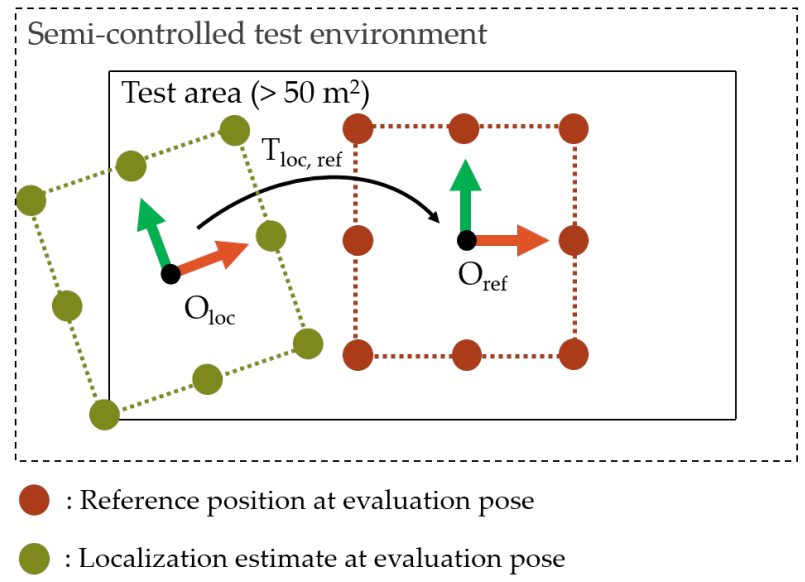

Figure 8. Determination of the transformation matrix $T_{r e f, l o c}$ between $O_{l o c}$ and $O_{r e f}$ with the Umeyama-Alignment [48].

Finally, the Experiment Data is recorded from the localization and reference data, while the ELT is passing the evaluation poses in sequence (3). When several localization systems are tested for the same Scenario, it is recommended to record the location estimates from multiple systems simultaneously for improving comparability, as suggested by Lymberopoulos et al. [7]. In case of manual guidance, it is recommended to monitor the ELT's current pose and provide the coordinates of the following evaluation pose. In case of using a robot, it is recommended that the robot obtains the data for its navigation from the reference system.

The output data of the Experiment Execution consists of the transformation matrix $T_{r e f, l o c}$ and a list of continuous location estimates and reference data with a synchronized timestamp. The transformation is applied to the localization data in the Performance Evaluation.

\subsection{Performance Evaluation ( $f$ )}

The Performance Evaluation aims to produce comparable results based on the Experiment Data and to analyze system behavior. The Performance Results consist of performance metrics and data for visualization. The functions of the Performance Evaluation are presented in Figure $9 f$. 


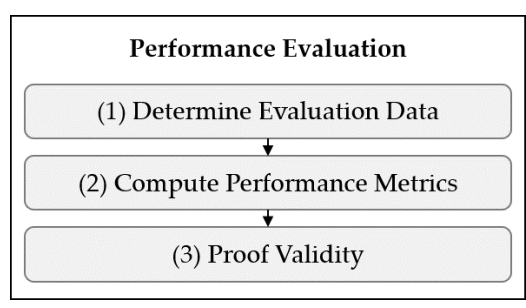

(f)

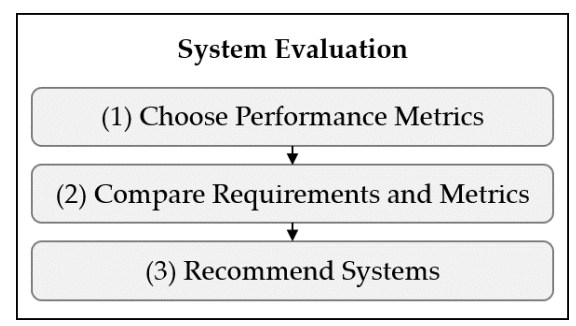

(g)

Figure 9. Functions of the module Performance Evaluation (f) and System Evaluation (g).

First, the Evaluation Data is determined (1). While the Experiment Data consists of the continuous data as measured in the experiment, the Evaluation Data is based on the aligned location estimate at the evaluation poses. The alignment of the continuous localization data is achieved by applying the transformation $T_{r e f, l o c}$. Then, the reference data is linearly interpolated to match the timestamp of the localization data. Finally, the data points corresponding to the position of the reference system closest to the defined evaluation pose are selected. Figure 10 shows the aligned and interpolated localization and reference data for an exemplary experiment. The left side shows a top view of the test area within the semicontrolled environment. Also shown are the evaluation poses $(0-4)$ with their tolerance $b$ and the reference and localization data, each defining a trajectory on the horizontal plane. A focused view of test point 2 is shown on the right. A valid data point requires that the reference trajectory passes through the tolerance area. Other tolerances related to the heading or velocity of the ELT may be considered. The data point is selected for the minimum difference $a_{m i n, t_{k}}$ between evaluation pose and reference data at the time $t_{k}$. The associated data point from the SUT is selected by the corresponding timestamp, resulting in the Evaluation Data.
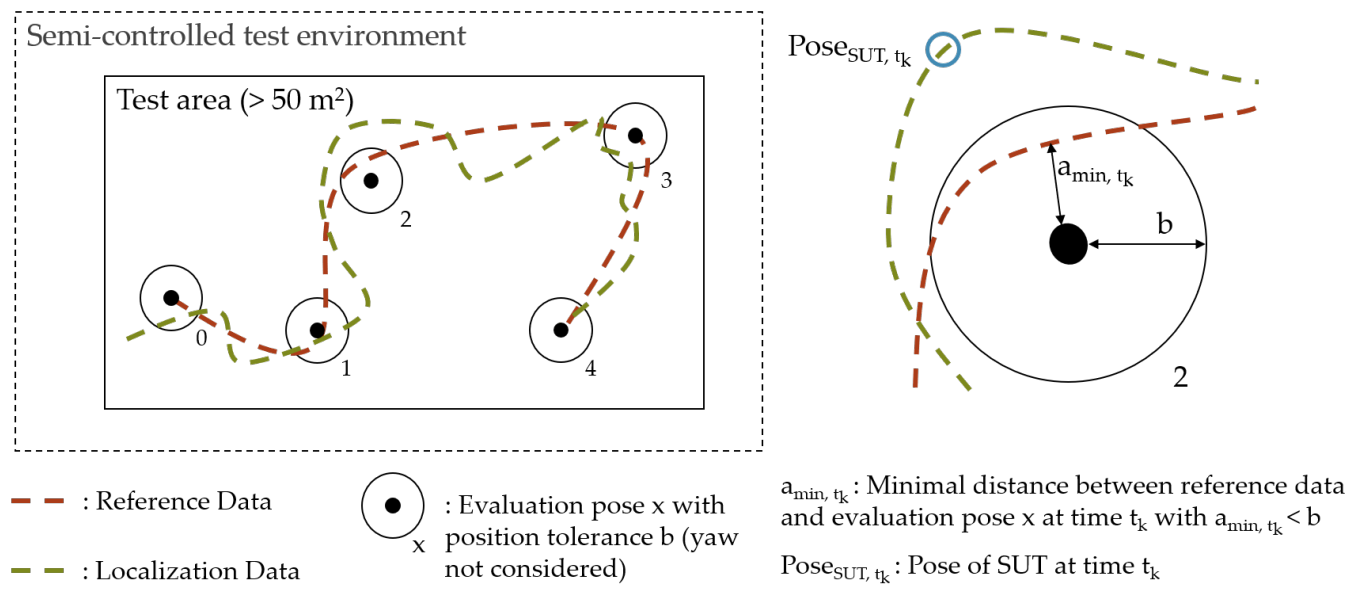

Figure 10. The left side shows a top view of the test area in a semi-controlled test environment, with evaluation poses, interpolated reference data, and aligned localization data. On the right side, a focused view of a test point is shown to illustrate the determination of the Evaluation Data.

Performance metrics are then calculated (2). The Performance Evaluation aims to provide a holistic overview of the system behaviour. Therefore, several metrics are calculated based on the error vectors. The 3D-error vector $\epsilon_{(x, y, z)}$ and the absolute 3D-error vector $\epsilon_{(|x|,|y|,|z|)}$ are determined by

$$
\begin{aligned}
\epsilon_{(x, y, z), i} & =\left(\hat{x}_{i}-x_{i}, \hat{y}_{i}-y_{i}, \hat{z}_{i}-z_{i}\right), \\
& \text { and } \\
\epsilon_{(|x|,|y|,|z|), i} & =\left(\left|\hat{x}_{i}-x_{i}\right|,\left|\hat{y}_{i}-y_{i}\right|,\left|\hat{z}_{i}-z_{i}\right|\right),
\end{aligned}
$$

whereby $\left(\hat{x}_{i}, \hat{y}_{i}, \hat{z}_{i}\right)$ denote a location estimate from the SUT and $\left(x_{i}, y_{i}, z_{i}\right)$ denote the corresponding data point from the reference system, with $i=1,2,3, . ., n$ and $n$ equals the 
amount of evaluation poses. The horizontal, vertical and spherical position error vectors are determined by computing the euclidian error of the respective components.

The heading error $\epsilon_{\text {yaw }}$ and the absolute heading error $\epsilon_{|y a w|}$ of the heading estimate $\hat{\psi}_{i}$ and the reference heading $\psi_{i}$ is determined by

$$
\begin{gathered}
\epsilon_{\text {yaw }, i}=\bmod \left(\left(\hat{\psi}_{i}+\pi\right), 2 \pi\right)-\pi, \\
\text { and } \\
\left.\epsilon_{\mid \text {yaw } \mid, i}=\mid \bmod \left(\hat{\psi}_{i}+\pi\right), 2 \pi\right)-\pi \mid,
\end{gathered}
$$

whereby $\bmod (a, b)$ elaborates the remainder of $a$ and $b$.

The following performance metrics are calculated from the vectors of the position error, the absolute position error, the horizontal, vertical, and spherical position error, and the heading error:

- Mean;

- $\quad$ Standard deviation;

- Median;

- RMSE;

- Variances of magnitudes;

- $\quad$ 95\%-percentile and percentiles related to $\sigma$-levels, as presented in Section 3.4

In practice, many things can go wrong during test and evaluation. For example, problems with data recording, clock synchronization, calibration, alignment determination or data processing are common. To ensure the validity of the T\&E results, the Experiment Data and the Performance Results must be carefully checked (3). Visualizations are recommended to gain an understanding of the system behavior. The repeatability or reproducibility of an experiment can be determined by comparing the Performance Results from various experiments. In addition, the impact of application-driven influences on the system performance can be examined.

\subsection{System Evaluation $(g)$}

Potortì et al. [5] criticize metrics defined in the ISO/IEC 18305 for being difficult for the system user to interpret and call for the exclusive use of the $95 \%$-percentile. Since applications of localization vary widely, it is not satisfactory for the system user to focus on only one metric. For instance, for customer tracking in a supermarket, it may be sufficient to consider the $93,3 \%$-percentile $(3 \sigma)$ with low position reliability requirements, while for a safety-critical application, such as an autonomous forklift, the percentile corresponding to the $6 \sigma$-level might be considered. To resolve this conflict, the TEE 4iLoc Framework provides a System Evaluation that focuses on the system user and is separate from the Performance Evaluation to support comprehensibility for system users without losing relevant information for developers and testers.

The System Evaluation is based on the Requirements defined in the Requirement Specification and on the Performance Results with the aim of determining the suitability of a localization system for a AUC. The Requirements consist of the description of absolute accuracy and confidence. The module's functions are shown in Figure 11g To determine the suitability of a system, the performance metrics corresponding to the requirements are first selected (1). The confidence level specified corresponds to the type of percentile to be considered. Then, the requirements and the performance metrics are compared (2). Finally, a system is considered suitable, if all 'must' requirements of an application are met (3). If 'shall' requirements are not met, the system is still recommended, but limitations are pointed out.

Finally, Figure 11 provides an overview of the TEE 4iLoc Framework, including the presented functions for each module. The framework enables the profound determination of a system's suitability for an application, based on application-driven test and evaluation in a semi-controlled environment. It is open to generic localization systems and focuses 
on the stakeholder requirements for T\&E by providing different function modules. The flexible definition of application-dependent scenarios addresses transferability, while the provision of a reference scenario aims at repeatability and reproducibility.

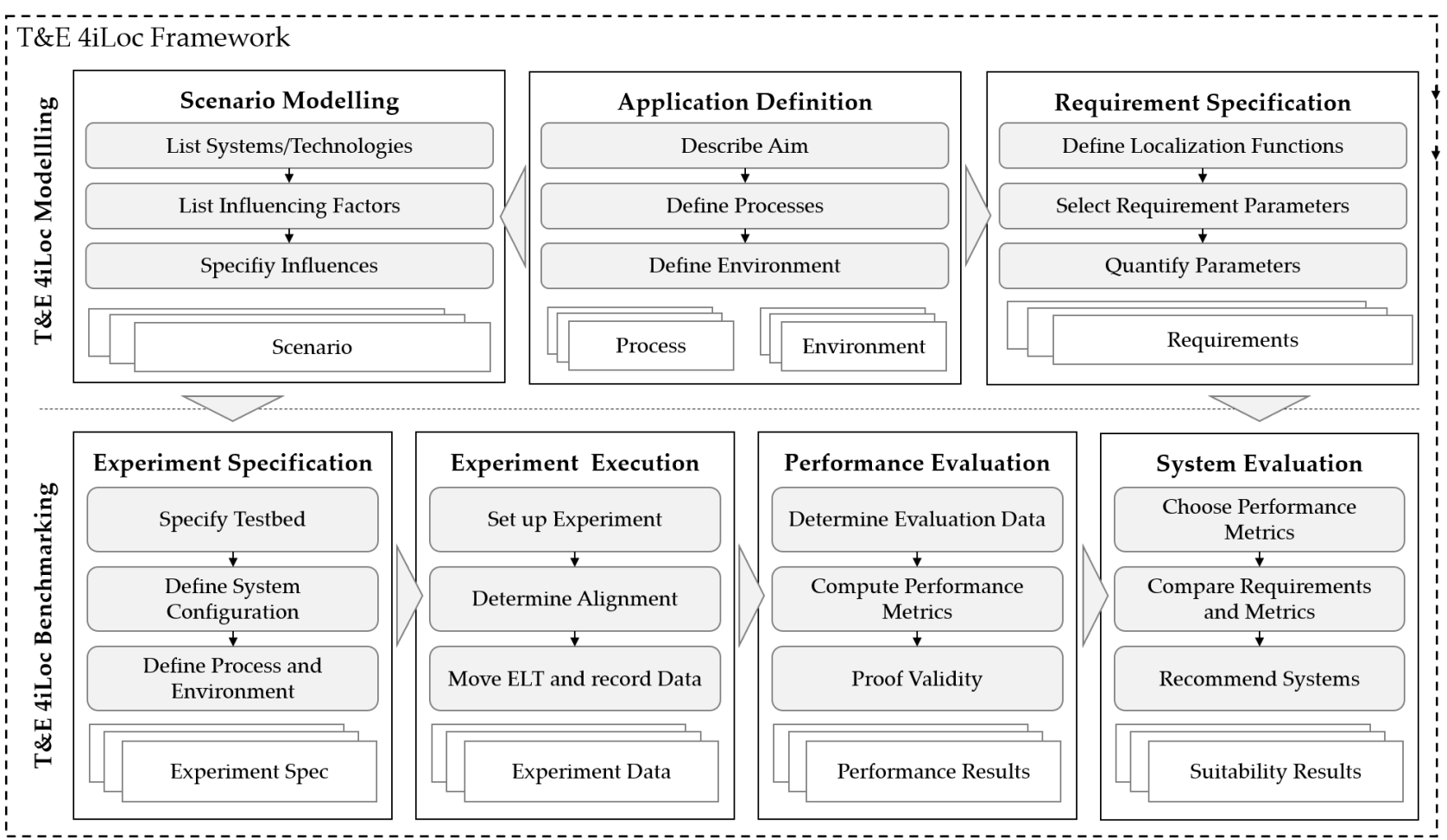

Figure 11. Overview of the TEE 4iLoc Framework, with its modules, functions and output data.

\section{Empirical Validation}

Meaningful test and evaluation implies, that localization systems of various technologies can be analyzed and compared for their performance and suitability for an application. In the following, the TEE 4iLoc Framework is used for test and evaluation to examine the framework's validity, by considering a logistics application.

In logistics processes, everything revolves around the movement of goods and commodities. They can only be moved effectively and efficiently if their position is known. Consequently, ILS are an essential tool for improving the understanding and control of processes related to material flow. On the one hand, a logistics application is considered for the empirical validation of the TEE 4iLoc Framework, since indoor localization systems are attributed an enormous potential for optimizing material flows. For example, Lee et al. [49] present an application for the tracking of assets, Reinke and Beinschob [50] for enabling Automated Guided Vehicles (AGV) and Macoir et al. [51] for automated inventory management in warehouses. On the other hand, warehouse environments are suitable to be modelled in semi-controlled test environments. In warehouses, the aim is to achieve a high ratio of usable space to floor area. The need for walls, e.g. for fire protection measures, leads to the division of a warehouse into large compartments. Special features in these compartments result from the provision of logistics systems. Test halls resemble warehouse compartments in their basic structure and the features of a warehouse relevant for localization systems can be modeled on a smaller scale.

In the following, a UWB-based, a camera-based, and a LiDAR-based ILS are examined for the application of AGVs in warehouse operation. The AUC is analyzed and modelled using the TEE 4iLoc Modelling Procedure. The performance and suitability of the systems are then determined based on empirical data from the TEE 4iLoc Benchmarking Procedure. The Experiment Data and the software script used for the performance evaluation are publicly available [52]. The Jupyter Notebook file [52] can be used for data comprehension. The test 
and evaluation procedure is described by explaining the execution of the framework's function modules.

\section{Application Definition}

The goal of the AUC 'Automated Guided Vehicle in warehouse operation' is to increase efficiency and safety by flexibly automatizing the material flow in warehouses. Navigation is one of the most essential component of a robot. It serves to find an efficient and collisionfree path. The task of navigation is commonly divided into global and local navigation. Global navigation aims at finding the approximate path to reach a certain goal, while local navigation focuses on robotics control [53]. For local navigation, the robot must to be aware of its surroundings, which is usually achieved by employing distance sensors. For efficient global navigation, the robot must know its absolute position and heading in the operating environment. In the following, 'Global navigation' is considered as a process for application-dependent test and evaluation. Nonetheless, many more processes can be defined to enable the efficient use of a AGV in warehouse operations, such as automatic picking and placement of goods or collision avoidance. As for the environment, a general warehouse is considered, characterized by shelving and conveyor systems, floor storage areas and areas for parking industrial trucks.

\section{Requirement Specification}

Identifying the aisle in which the robot is located is considered the critical localization function for the process of 'global navigation'. As a AGV is a floor-bound vehicle, the absolute position must be known in the horizontal plane. To quantify this parameter, the dimensions of the robot in an aisle are considered as shown in Figure 12. The aisle width is $1,50 \mathrm{~m}$, the robot's width is $0,6 \mathrm{~m}$ and the shelf depth is $0,5 \mathrm{~m}$. To avoid collisions, there is an additional tolerance distance between the AGV and shelves of $0,2 \mathrm{~m}$, resulting in a guidance width of $1,1 \mathrm{~m}$. Even if the robot travels close to the sides of the aisle, the position of the center of the robot must be determined so that it is within the correct aisle. Requirements for the horizontal position accuracy are calculated as the sum of the shelf depth, the tolerance width, and half of the robot's width, resulting in a horizontal position accuracy requirement of $0,75 \mathrm{~m}$.

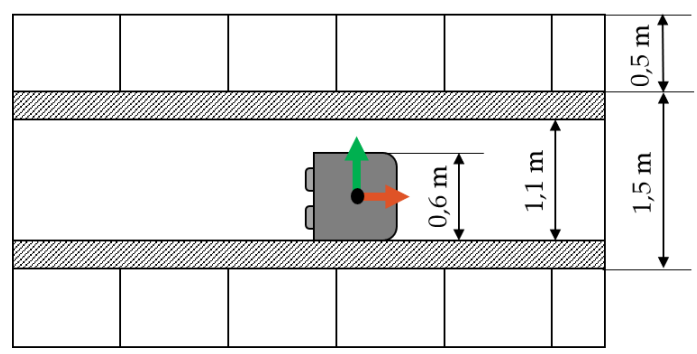

Figure 12. Dimensions of a AGV in an aisle for the quantification of performance requirements.

In addition, the robot's heading information can be used to improve global navigation. Hence, the heading is considered a 'shall' requirement. For the process of global navigation, the robot's heading is mainly relevant to identify the side of the aisle it is facing. The robot is assumed to be directed in one of the two directions of the aisle with a tolerance of $\pm 30^{\circ}$. Consequently, the heading requirement for identifying the correct side is $\pm 60^{\circ}$. Unlike for local path planning, safety is less of a concern for global path planning. Therefore, a moderate confidence level of $4 \sigma$, i.e. $99,38 \%$ is chosen. The requirements for the process 'global navigation' are summarized in Table 4. 
Table 4. Performance requirements for the process 'global navigation'.

\begin{tabular}{cccc}
\hline Parameter & Absolute Accuracy & Shall/Must & Confidence \\
\hline Horizontal position & $<0,75 \mathrm{~m}$ & Must & $99,38 \%$ \\
Heading & $<60^{\circ}$ & Shall & $99,38 \%$ \\
\hline
\end{tabular}

Scenario Modelling

To enable the specification of a meaningful experiment, it is necessary to derive an application-dependent scenario. First, the considered localization systems, i.e. the UWB ILS, LiDAR-based ILS, and camera-based ILS, and their application-dependent influencing factors need to be considered. The location of the LiDAR and the camera is determined, by comparing the sensor data with a previously recorded map of the environment [54]. Consequently, the systems' performance is influenced by the objects in the environment. RF-based localization systems, such as UWB are generally prone to errors in so-called None-Line-of-Sight (NLOS) conditions. NLOS occurs when the direct line between the transmitting and receiving nodes of a signal is blocked. The material and structure of the occluding objects are important because they influence the signal transmission and reflection [55]. Hence, objects in the environment are considered as a significant influencing factor for defining an application-dependent scenario. To resemble a warehouse environment, shelves with goods of different materials, vehicles, and other logistics equipment are considered. Common warehouse dynamics are mirrored by slightly altering the layout after map recording. Another environmental influence on the performance of the camera-based ILS is the lighting. A mixture of daylight and artificial light is chosen as another application-dependent influence to reflect a typical warehouse environment.

For process influences, the ELT type, the motion, and the path are considered. To reflect the motion of a AGV, the ELT type 'robot' is selected. Especially in mixed traffic environments, such as a typical warehouse, robots move at slow velocity. Considering the path, a AGV moves freely on the horizontal plane of a warehouse. For the test scenario, it is required for the robot to move through an aisle. Table 5 summarizes the applicationdependent scenario for the AUC.

Table 5. Application-dependent Scenario for a AGV in warehouse operation.

\begin{tabular}{c|ccc|cc}
\hline & \multicolumn{3}{|c}{ Process Influences } & \multicolumn{2}{c}{ Environment Influences } \\
\hline Factors & $\begin{array}{c}\text { Entity to be } \\
\text { Localized }\end{array}$ & Motion & Path & $\begin{array}{c}\text { Static } \\
\text { Objects }\end{array}$ & $\begin{array}{c}\text { Lighting } \\
\text { Conditions }\end{array}$ \\
\hline Scenario & Robot & $\begin{array}{c}\text { Slow velocity; } \\
\text { acceleration/ } \\
\text { deceleration }\end{array}$ & $\begin{array}{c}\text { curves on } \\
\text { horizontal } \\
\text { plane; } \\
\text { driving } \\
\text { through aisle }\end{array}$ & $\begin{array}{c}\text { Shelves; } \\
\text { vehicles; } \\
\text { logistics } \\
\text { equipment } \\
\text { (altered after } \\
\text { mapping) }\end{array}$ & $\begin{array}{c}\text { Daylight; } \\
\text { artificial } \\
\text { Light }\end{array}$ \\
\hline
\end{tabular}

Experiment Specification

The testbed at the Institute for Technical Logistics at Hamburg University of Technology is considered a semi-controlled test environment. By providing a rectangular test area of $63 \mathrm{~m}^{2}$ the framework requirements regarding the size of the test area are met. The environment can be equipped with various objects, such as shelves, pallets, industrial trucks, and other logistics equipment. The testbed features an optical passive motion capture system that enables the determination of 6-DoF reference pose data, with an absolute accuracy of around $1 \mathrm{~mm}$. Sensors and reflectors are mounted on a TurtleBot2 robotic platform. (Figure 13a). The robot is automatically controlled based on the localization data from the reference system. 


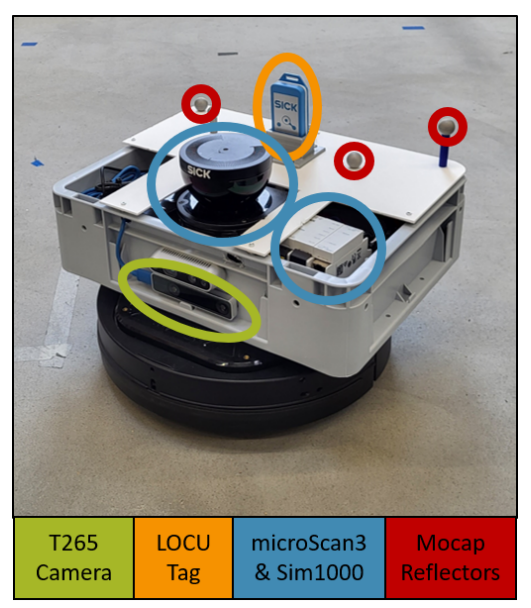

(a)

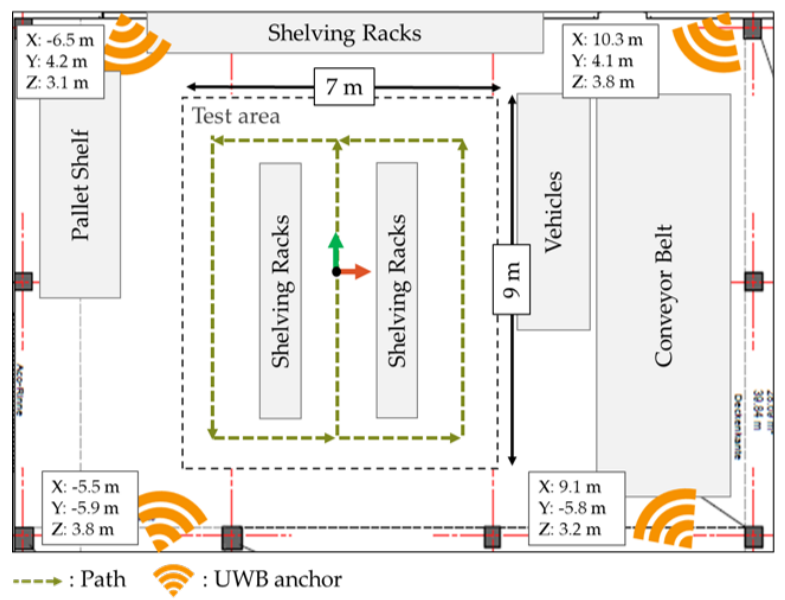

(b)

Figure 13. (a) Turtlebot2 carrying the localization sensors and motion capture reflectors. (b) Schematic overview of the Experiment Spec.

The UWB ILS (LOCU [56], SICK AG) consists of four anchors and a localization tag. The anchors are uniformly distributed, avoiding symmetries. '2D-Mode' is enabled to improve the system's horizontal position accuracy by limiting the DoF. The LiDAR system consists of a multi-layer safety LiDAR scanner (microScan3 [57], SICK AG) for emission and detection of laser pulses, a control unit (SIM1000 [58], SICK AG), and localization software (LiDAR-LOC [59], SICK AG). The pose of the sensor is determined by comparing scan points with a pre-recorded map applying a particle filter [60]. The field of view of the laser scanner is $270^{\circ}$ the localization is supported by an internal Inertial Measurement Unit (IMU). Finally, the localization of the Intel RealSense T265 Tracking Camera [61] is achieved based on a feature-based visual Simultaneous Localization and Mapping (vSLAM) algorithm [62]. The stereo camera is likewise equipped with an IMU and allows identification of loop closures. All systems are deployed according to the installation instructions.

Figure 13b gives a schematic overview of the Experiment Spec. The test area is surrounded by typical warehouse objects, such as a pallet shelf, industrial trucks and a conveyor belt. Two rows of shelving racks are placed inside the test area to build an aisle. 73 evaluation poses are sampled on a grid with $1 \mathrm{~m}$ grid length, to guide the robot multiple times through the aisle and around the shelves. The resulting path is depicted in Figure 13b.

\section{Experiment Execution}

The environment and the SUT are set up according to the Experiment Spec. A photo of the environment at the Institute for Technical Logistics is shown in Figure 14a. The shelves on the test area are imitated by objects of different materials, resulting in NLOS conditions for the UWB ILS. The reference system is calibrated and synchronized to the localization systems via Precision Time Protocol (PTP) [63] with an offset of less than $0.5 \mathrm{~ms}$. The reference system is set up, to accurately locate the origins of the devices on the robot. The Umeyama-Alignments for the SUT are then determined based on the measurements from the localization and reference data at nine uniformly distributed positions on the test area. Maps of the environment are recorded for the LiDAR and camera-based ILS. The recorded contour map from the LiDAR ILS is shown in Figure 14b. Then, the sequence of four of the static objects is altered, to replicate realistic layout changes in a real warehouse environment.

Finally, the experiment is executed. The localization data is recorded simultaneously from the three SUT and the reference system. The Robot Operating System (ROS) [64] is used to control the robot and record the data. 


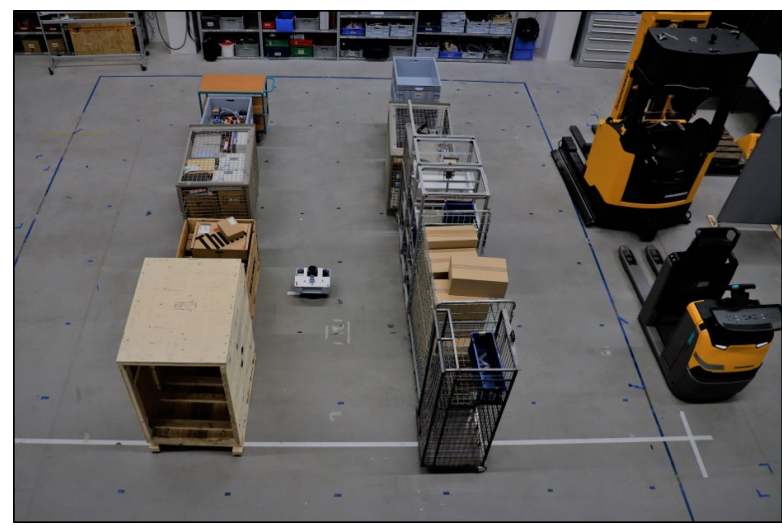

(a)

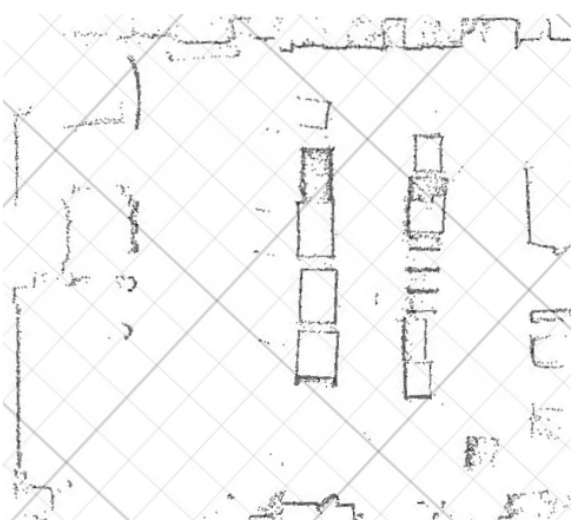

(b)

Figure 14. (a) Setup of the environment at the Institute for Technical Logistics. (b) Recorded map from the LiDAR ILS. The grid with a grid length of $1 \mathrm{~m}$ is aligned with the map coordinate system.

Performance Evaluation

The Evaluation Data are computed according to the methods presented in Section 3.8. The Performance Results are presented below. The goal is not to provide an exhaustive analysis, but to demonstrate approaches and the need for studying the behavior of the systems. Due to their relevance to the AUC, the focus of the evaluation is on the horizontal components. The performance metrics related to the 2D-error components $(x, y, y a w)$ are presented in Table 6.

Table 6. Overview of performance metrics. Metrics referred to in the text are marked bold.

\begin{tabular}{|c|c|c|c|c|c|c|c|}
\hline & Mean & $\begin{array}{l}\text { Std. De- } \\
\text { viation }\end{array}$ & Median & RMSE & $\begin{array}{l}\text { Variance } \\
\text { of Magni- } \\
\text { tude }\end{array}$ & $\begin{array}{c}95 \%- \\
\text { percentile }\end{array}$ & $\begin{array}{c}99,38 \%- \\
\text { percentile } \\
(4 \sigma)\end{array}$ \\
\hline \multicolumn{8}{|c|}{ SICK LOCU UWB } \\
\hline$\epsilon_{x}$ & 0,003 & 0,174 & 0,006 & 0,001 & 0,008 & 0,268 & 0,335 \\
\hline$\epsilon_{y}$ & 0,046 & 0,259 & 0,054 & 0,154 & 0,022 & 0,441 & 0,525 \\
\hline$\epsilon_{|x|}$ & 0,149 & 0,090 & 0,138 & 1,637 & 0,008 & 0,291 & 0,366 \\
\hline$\epsilon_{|y|}$ & 0,217 & 0,148 & 0,224 & 3,482 & 0,022 & 0,469 & 0,539 \\
\hline$\epsilon_{\text {horizontal }}$ & 0,283 & 0,138 & 0,270 & 5,926 & 0,019 & 0,530 & 0,582 \\
\hline \multicolumn{8}{|c|}{ "SICK LiDAR-LOC } \\
\hline$\epsilon_{x}$ & $-0,010$ & 0,062 & $-0,004$ & 0,007 & 0,002 & 0,090 & 0,111 \\
\hline$\epsilon_{y}$ & 0,009 & 0,064 & 0,029 & 0,006 & 0,001 & 0,090 & 0,112 \\
\hline$\epsilon_{|x|}$ & 0,049 & 0,039 & 0,042 & 0,180 & 0,002 & 0,115 & 0,154 \\
\hline$\epsilon_{|y|}$ & 0,060 & 0,024 & 0,061 & 0,264 & 0,001 & 0,094 & 0,112 \\
\hline$\epsilon_{\text {horizontal }}$ & 0,085 & 0,030 & 0,082 & 0,532 & 0,001 & 0,131 & 0,166 \\
\hline$\epsilon_{\text {yaw }}$ & $-2,209$ & 1,819 & $-2,155$ & 361,091 & 2,611 & 0,615 & 0,890 \\
\hline$\epsilon_{\mid \text {yaw } \mid}$ & 2,362 & 1,616 & 2,155 & 412,785 & 2,611 & 3,826 & 8,772 \\
\hline \multicolumn{8}{|c|}{ Intel RealSense T265 } \\
\hline$\epsilon_{x}$ & 0,239 & 0,288 & 0,188 & 4,233 & 0,061 & 0,846 & 0,994 \\
\hline$\epsilon_{y}$ & 0,633 & 1,101 & 0,363 & 29,662 & 1,163 & 3,114 & 4,879 \\
\hline$\epsilon_{|x|}$ & 0,282 & 0,247 & 0,193 & 5,872 & 0,061 & 0,846 & 1,014 \\
\hline$\epsilon_{|y|}$ & 0,671 & 1,078 & 0,374 & 33,312 & 1,163 & 3,114 & 4,880 \\
\hline$\epsilon_{\text {horizontal }}$ & 0,817 & 1,042 & 0,537 & 49,391 & 1,086 & 3,133 & 4,881 \\
\hline$\epsilon_{\text {yaw }}$ & $-0,311$ & 2,072 & 0,011 & 7,168 & 1,606 & 2,320 & 3,496 \\
\hline$\epsilon_{|y a w|}$ & 1,668 & 1,267 & 1,475 & 205,884 & 1,606 & 4,203 & 4,714 \\
\hline
\end{tabular}

$\overline{\epsilon_{x}}$ : Error vector $\mathrm{x} ; \epsilon_{y}:$ Error vector $\mathrm{y} ; \epsilon_{|x|}:$ Absolute error vector $\mathrm{x} ; \epsilon_{|y|}:$ Absolute error vector $\mathrm{y} ; \epsilon_{\text {horizontal }}:$ Error vector horizontal; $\epsilon_{\text {yaw }}$ : Error vector heading; $\epsilon_{\mid \text {yaw } \mid}$ : Absolute error vector heading.

Length in [m]; angle in [ deg] 
In the following, selected metrics are discussed for the LiDAR-based system. The low mean value and RMSE of the error vector $\epsilon_{x}$ and $\epsilon_{y}$, compared to the respective absolute error vectors, indicate good coordinate alignment and rather low location-dependent inaccuracies. The absolute value of the mean heading error $\epsilon_{\text {yaw }}$ is rather high compared to the corresponding standard deviation. A possible reason for this systematic error may be a misalignment of the coordinate axes between the LiDAR sensor and the motion capture reflectors. To compensate for this, the mean of $\epsilon_{\text {yaw }}$ could simply be removed as a bias. However, this would also lead to the neglection of location-dependent errors. For a system user, the high percentiles (e.g. the 95\%-percentile or high sigma levels) of the horizontal position error $\epsilon_{\text {horizontal }}$ and the absolute heading error $\epsilon_{\mid \text {yaw }}$ are of particular importance.

In addition, several graphs are shown in Figure 15. The trajectories from Figure 15a are based on the aligned position data of the localization systems, while the other graphs are based on the discrete evaluation data. The trajectory of the camera-based ILS shows significant deviations from the evaluation poses. This is reflected as a leap in the horizontal error graph (15b). The heading error is not particularly affected by the leaps of the position error. A possible reason for the behavior could be the relocalization of the system in the previously recorded map due to altering the environment after map recording. The position data of the UWB ILS is characterized by a deviation to the center of the test area (15a) and comparatively high dispersion (15d). However, there are no major outliers. With the exception of one outlier in the heading estimate, the LiDAR ILS shows reliably good system behavior in terms of horizontal and heading error.

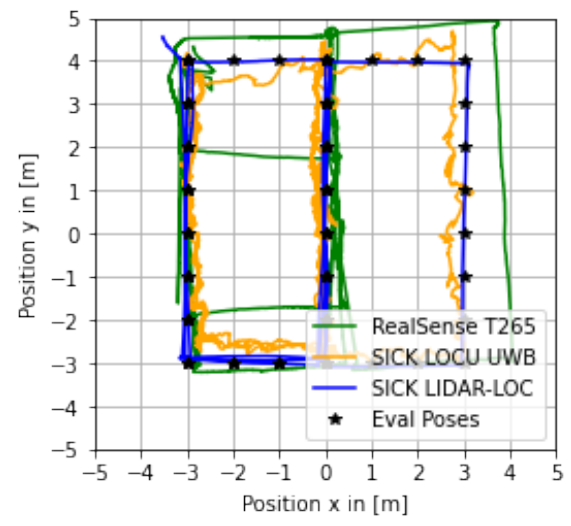

(a)

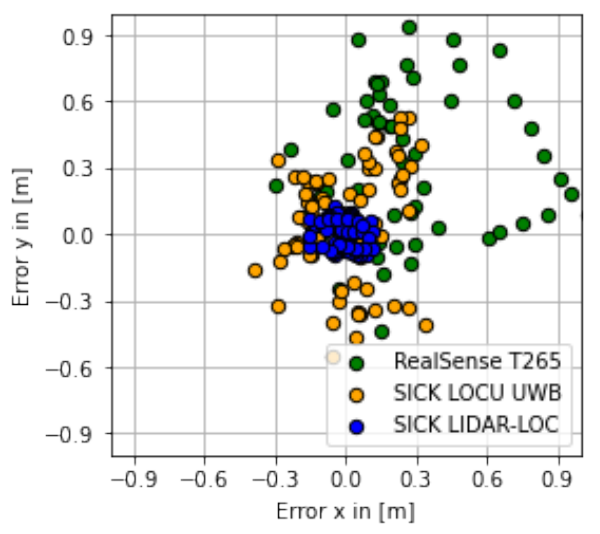

(d)

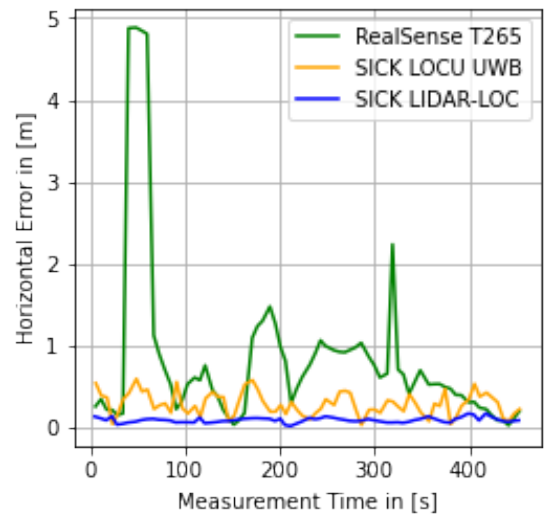

(b)

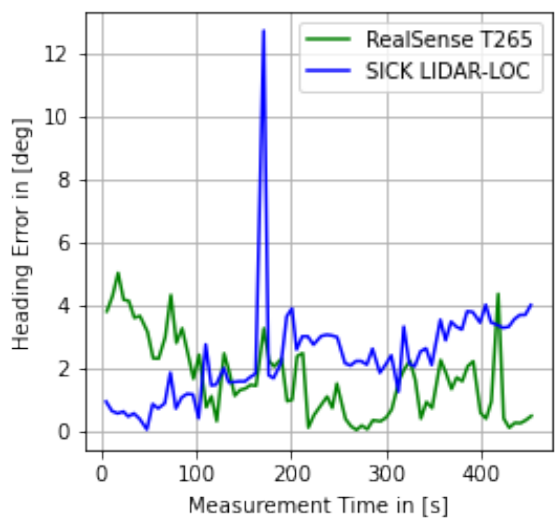

(e)

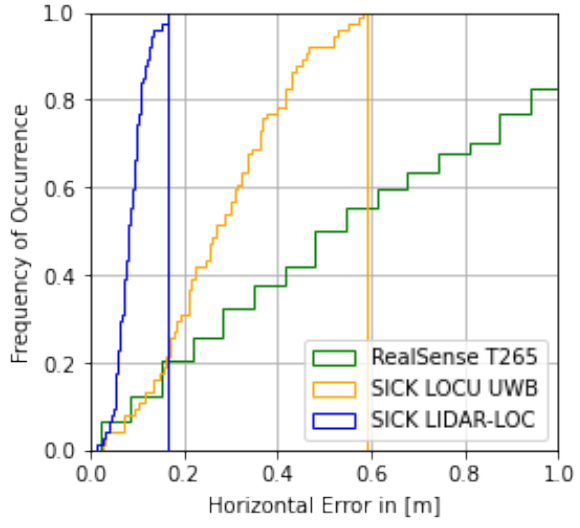

(c)

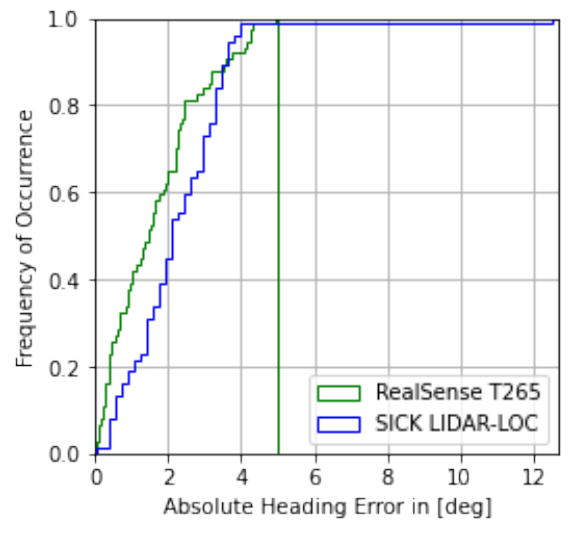

(f)

Figure 15. (a) Trajectories based on continuous position estimates. (b) Horizontal error over measurement time. (c) cumulative distribution histogram of the horizontal error. (d) Error scatter. (e) Heading error over measurement time. (f) Cumulative distribution histogram of the absolute heading error. 
System Evaluation

Finally, the derived performance requirements are matched with the calculated metrics to determine the suitability of a localization system for the process 'global navigation'. As previously discussed, the relevant performance requirements are the $4 \sigma$-percentiles of the horizontal position error $\epsilon_{\text {horizontal }}$ ('must') and the absolute heading error $\epsilon_{\text {horizontal }}$ ('shall'). Table 7 provides a comparison between the requirements and metrics, where the red font color indicates that a requirement is not met. The 'must'-requirement is met by both the UWB and the LiDAR-based ILS. Hence, both systems are considered suitable for 'global navigation'. Since the UWB ILS does not provide heading information, the horizontal position and absolute heading error requirements are only met by the LiDAR system. However, both systems are generally considered suitable for the AUC to enable 'global navigation'. For the camera-based ILS, the requirements regarding the horizontal position error is not met.

Table 7. Comparison of requirements and respective performance metrics of the SUT for the process 'global navigation'.

\begin{tabular}{cc|ccc}
\hline Parameter & Requirement & $\begin{array}{c}\text { SICK LOCU } \\
\text { UWB }\end{array}$ & $\begin{array}{c}\text { SICK } \\
\text { LiDAR-LOC }\end{array}$ & $\begin{array}{c}\text { Intel RealSense } \\
\text { T265 Tracking } \\
\text { Camera }\end{array}$ \\
\hline $\begin{array}{c}\text { Horizontal } \\
\text { position error }\end{array}$ & $\begin{array}{c}<0,75 \mathrm{~m} \\
(\text { must, } 4 \sigma \text {-level) }\end{array}$ & $0,58 \mathrm{~m}$ & $0,17 \mathrm{~m}$ & $4,88 \mathrm{~m}$ \\
\hline $\begin{array}{c}<60^{\circ} \\
\text { Heading error }\end{array}$ & - & $8,77^{\circ}$ & $4,71^{\circ}$ \\
\hline
\end{tabular}

The TEE 4iLoc Framework was designed with the aim to increase meaningfulness of $T \& E$ in semi-controlled environments. Requirements were specified and test scenarios designed for the application 'AGVs in warehouse operation'. Application-dependent test and evaluation was performed, resulting in several performance metrics for comparison between systems and the determination of the systems' suitability. Based on the empirical validation, two aspects are highlighted to demonstrate the relevance of the presented methodology. First, the relevance of application-driven test and evaluation is shown by comparison to results from the literature. In an experiment presented by Hausamann et al. [65], analysis of the absolute position accuracy of the RealSense T265 Tracking Camera based on continuous position data yielded a mean horizontal error of around $0,4 \mathrm{~m}$. This is in contrast to a mean error of $0,8 \mathrm{~m}$ for application-dependent testing in this work. The data presented by Hausamann et al. [65] was gathered by tracking a person on a significantly smaller test area, without the consideration of aisles or alterations of the environment after map recording. Hence, it may be misleading to consider the test and evaluation results for the application 'AGV in warehouse operation'. The second aspect consists in the need of thoroughly examining the experiment data before drawing final conclusions. Performance metrics are absolutely necessary to compare systems and determine their suitability for an application. However, important characteristics of a system remain hidden if only one metric is looked at. By providing multiple metrics and graphical data representations, possible reasons for localization errors can be identified. Some of these may be relevant for the application, while others should be disregarded.

\section{Discussion and Conclusion}

High heterogeneity of indoor localization technologies, applications, and influences pose multiple challenges for test and evaluation of ILS. By comparing test and evaluation of ILS in practice, the need for methodological approaches becomes evident. Methodologies exist, but they do not fully satisfy stakeholders' requirements. The field of tension of stakeholder requirements was presented in Section 2.1, consisting of feasibility, reproducibility, comparability, transferability, and comprehensibility. In this work, the TEE 4iLoc Framework was introduced - A methodology for black-box, system-level test and evaluation 
in semi-controlled environments. Similar to the existing methodologies, the TEE 4 iLoc Framework is based on a system-level and black-box approach. The main benefits and disadvantages arise from the focus on semi-controlled test environments. By using the TEE 4iLoc Framework, the drawbacks of low feasibility, comparability, and reproducibility in building-wide testing can be overcome. On the other hand, building-wide tests have the advantage that the results are highly transferable to real-world applications. To produce results not only meaningful for system developers but also system user, the TEE $4 i L O C$ Framework is designed to be application-driven. Hence, consideration of an application or application domain affects both the test and the evaluation of localization systems. Comprehensibility is addressed by providing a modular and process-oriented procedure. The use of the methodology for empirical validation has demonstrated its feasibility. Comparability between systems has been demonstrated for benchmarking the same scenario in the same testbed. Additional experiments are planned to further examine the reproducibility, comparability, and transferability of the test and evaluation results.

Different limitation exist. (1) To achieve meaningful results for T\&E in semi-controlled test environments, the AUC and the SUT must be suitable. For example, meaningful test and evaluation of workers in an underground mine based on ultrasonic ILS, as presented by [66], can hardly be achieved. (2) The spatial extent can significantly affect the absolute accuracy of a ILS. Further examination is required, to derive conclusion and potentially metrics for spatial scaling effects. (3) So far, only accuracy metrics that are based on the absolute localization error at discrete evaluation poses are considered. Although other metrics are currently not integrated in the TEE 4iLoc Framework, the determination of additional accuracy metrics, such as the repeatability when approaching the same pose several times, is possible. Other metrics, such as Fréchet distance [67] or relative position error [23] can be determined based on the continuous evaluation data. In addition, metrics such as the position error from the direction of the ELT or the velocity-dependent heading error can be determined to analyze system behavior and to test validity. (4) For a holistic evaluation the consideration of many more factors, such as the sensor weight, maintenance costs or the system's integrity are essential. An overview on possible user requirements is given by Mautz [4].

A web-based application to support systematic test and evaluation using the TEE 4iLoc Framework is currently under development and will be released in the near future. Besides the usage of the TEE 4iLoc Framework in empirical test and evaluation it is conceivable to adapt the methodology for approaches, such 'simulated data', 'common datasets' or 'indoor localization competitions' as presented in Section 2.2. Adoption or adaption of the TEE 4iLoc Framework in industry and research are encouraged to increase meaningfulness of test and evaluation results. This is essential to support the application-driven research and development of ILS, increase market transparency, thus, advancing the practical application of ILS.

Author Contributions: Conceptualization, J.S., J.H.; methodology, J.S., J.H.,J.K.; software, J.S.; validation, J.S.; formal analysis, J.S.; investigation, J.S. ; resources, J.S.; data curation, J.S.; writing-original draft preparation, J.S.; writing—review and editing, J.H.J.K; visualization, J.S.; supervision, J.H.,J.K.; project administration, J.K..; funding acquisition, J.K.; All authors have read and agreed to the published version of the manuscript.

Funding: This work is supported by internal funding from Hamburg University of Technology.

Institutional Review Board Statement: Not applicable.

Informed Consent Statement: Not applicable.

Data Availability Statement: The localization data, the reference data, and the software script used for the empirical validation are provided on GitLab in a publicly available repository [52].

Conflicts of Interest: The authors declare no conflict of interest. 


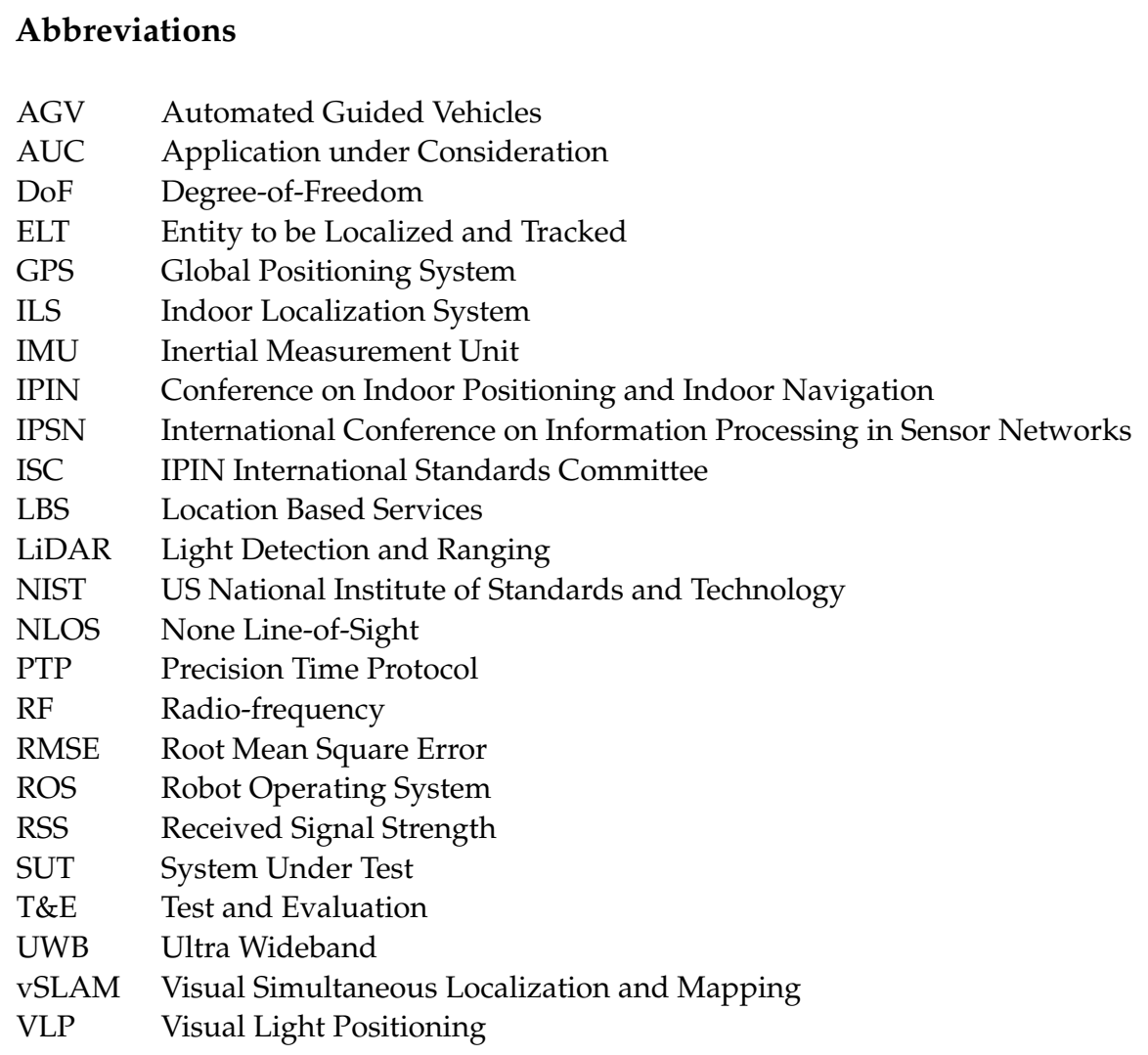

\section{Appendix A. Overview of Publications from IPIN 2021 with Empirical T\&E of the Absolute Accuracy}

Table A1 provides an overview of the 33 papers from IPIN conference 2021 in which empirical test and evaluation of absolute accuracy was performed. The system-type is divided into VLP, RF, magnetic, acoustic and multiple, depending on the underlying technology of the localization system. The type of ground truth is divided into reference system and other methods. The test environment is divided into hall, building, outdoor, room, small space and mixed (indoor and outdoor). 
Table A1. Overview of the 33 publications from IPIN conference 2021, in which empirical test and evaluation of the absolute position accuracy was carried out.

\begin{tabular}{|c|c|c|c|}
\hline Paper title & System-type & Ground Truth & Environment \\
\hline A Cellular Approach for Large Scale, Machine Learning Based Visible Light Positioning Solutions [19] & VLP & Reference System & Hall \\
\hline Accurate Multi-Zone UWB TDOA Localization utilizing Cascaded Wireless Clock Synchronization [68] & RF & Reference System & Building \\
\hline Periodic Extended Kalman Filter to Estimate Rowing Motion Indoors Using a Wearable Ultra-Wideband Ranging Positioning System [69] & RF & Reference System & Hall \\
\hline Foot-mounted INS for Resilient Real-time Positioning of Soldiers in Non-collaborative Indoor Surroundings [70] & Multiple & Other & Mixed \\
\hline Quantifying the Degradation of Radio Maps in Wi-Fi Fingerprinting [71] & RF & Other & Building \\
\hline Measuring Uncertainty in Signal Fingerprinting with Gaussian Processes Going Deep [72] & RF & Reference System & Building \\
\hline Analysis of IMU and GNSS Data Provided by Xiaomi 8 Smartphone [73] & Multiple & Other & Outdoor \\
\hline $\begin{array}{l}\text { Evolutionary-Inspired Strategy for Particle Distribution Optimization in Auxiliary Particle Filtering Algorithm Based Indoor Position- } \\
\text { ing [22] }\end{array}$ & RF & Other & Hall \\
\hline Comparing and Evaluating Indoor Positioning Techniques [74] & Multiple & Other & Room \\
\hline Indoor Positioning Using the OpenHPS Framework [75] & Multiple & Other & Building \\
\hline Anchor Pair Selection for Error Correction in Time Difference of Arrival (TDoA) Ultra Wideband (UWB) Positioning Systems [20] & RF & Reference System & Hall \\
\hline On the Use of Lookahead to Improve Wi-Fi Fingerprinting Indoor Localization Accuracy [76] & RF & Other & Building \\
\hline RAD-GAN: Radio Map Anomaly Detection for Fingerprint Indoor Positioning with GAN [77] & RF & Other & Building \\
\hline Multipath-Resilient Unsynchronized 2.4 GHz ISM-Band RF Positioning Using Coherent Echos [78] & RF & Reference System & Mixed \\
\hline Comparative Study of Different BLE Fingerprint Reconstruction Techniques [79] & RF & Other & Building \\
\hline Finding Optimal BLE Configuration for Indoor Positioning with Consumption Restrictions [80] & RF & Other & Building \\
\hline MM-Loc: Cross-sensor Indoor Smartphone Location Tracking using Multimodal Deep Neural Networks [81] & Multiple & Other & Building \\
\hline Experimental Investigation of 5G Positioning Performance Using a mmWave Measurement Setup [21] & RF & Other & Hall \\
\hline Magnetic Mapping for Robot Navigation in Indoor Environments [83] & Magnetic & Reference System & Hall \\
\hline Two Efficient and Easy-to-Use NLOS Mitigation Solutions to Indoor 3-D AOA-Based Localization [84] & Acoustic & Other & Hall \\
\hline POUCET: A Multi-Technology Indoor Positioning Solution for Firefighters and Soldiers [85] & Multiple & Other & Mixed \\
\hline Adaptive procedure for indoor localization using LoRa devices [86] & RF & Other & Hall \\
\hline Multidimensional In- and Outdoor Pedestrian Tracking using OpenStreetMap Data [87] & Multiple & Other & Building \\
\hline Indoor Localization Method For a Microphone Using a Single Speaker [88] & Acoustic & Other & Room \\
\hline Short-Time and Adaptive Controllable Spot Communication Using COTS Speaker [89] & Acoustic & Other & Small Space \\
\hline $\begin{array}{l}\text { Environment-Aware RSSI Based Positioning Algorithm for Random Angle Interference Cancellation in Visible Light Positioning } \\
\text { System [90] }\end{array}$ & VLP & Reference System & Room \\
\hline Evaluation of Non-Intrusive Positioning for Public Environments [91] & RF & Reference System & Building \\
\hline $\begin{array}{l}\text { Topology Construction Based on Indoor Crowdsourcing Data using Manifold Learning: Evaluation of Algorithms and Key Parame- } \\
\text { ters [92] }\end{array}$ & RF & Other & Building \\
\hline Smartphone Positioning Using an Ambient Light Sensor and Reflected Visible Light [93] & VLP & Other & Small Space \\
\hline Combined ADS-B and GNSS Indoor Localization [94] & RF & Other & Mixed \\
\hline Positioning Android Devices in Large Indoor Spaces and Transitioning to Outdoors by Sensor Fusion [95] & Multiple & Other & Building \\
\hline ProxyFAUG: Proximity-based Fingerprint Augmentation [96] & RF & Reference System & Outdoor \\
\hline
\end{tabular}




\section{Appendix B. Overview of the Terminology of the TEE 4iLoc Framework}

An overview of the terminology used in the TEE 4iLoc Framework is provided in Table A2.

Table A2. Terminology of the TEE 4iLoc Framework.

\begin{tabular}{|c|c|}
\hline Terminology & Explanation \\
\hline Alignment & Process for converting a SUT coordinate system to the reference coordinate system. \\
\hline Absolute Localization & $\begin{array}{l}\text { Location referring to a large-scale reference. In contrast, relative localization depends } \\
\text { on a local frame of reference. }\end{array}$ \\
\hline Application-driven influencing factors & $\begin{array}{l}\text { Parameters that are expected to influence the performance of a SUT. They can be derived } \\
\text { from the AUC and describe influences with a practical character. }\end{array}$ \\
\hline Application-dependent T\&E & Test and evaluation procedure in which a AUC affects both the test and the evaluation. \\
\hline Application under Consideration (AUC) & $\begin{array}{l}\text { The regarded application or application domain for which a SUT is to be tested and } \\
\text { evaluated. }\end{array}$ \\
\hline Environment Specification & Description of the configuration of a test facility for test and evaluation. \\
\hline Benchmark & Procedure for executing and evaluating an experiment. \\
\hline Configuration & A set of parameters that describe the environment or system for an experiment. \\
\hline Entity to be Localized/Tracked (ELT) & $\begin{array}{l}\text { Person, object, vehicle or robot, whose location is to be determined for the AUC or } \\
\text { experiment. }\end{array}$ \\
\hline Evaluation pose & Pose or position at which the accuracy of the SUT is evaluated. \\
\hline Evaluation data & $\begin{array}{l}\text { Timestamped and aligned pairs of location data from the SUT and the reference system } \\
\text { at the evaluation poses. }\end{array}$ \\
\hline Experiment & Physical execution of a Scenario. \\
\hline Experiment data & Continuous location data from the SUT and the reference system. \\
\hline Experiment setup & Configuration of the SUT and the environment according to an Experiment Spec \\
\hline Experiment Spec & Testbed-dependent description of an experiment. \\
\hline Test facility & Building with a semi-controlled test environment. \\
\hline Localization data & Timestamped position and heading data. \\
\hline Location estimate & Timestamped position and heading data provided by the SUT. \\
\hline Metrics & Indicator of performance for the SUT. \\
\hline Function module & A set of instructions that serve a higher-level purpose. \\
\hline Requirements & Set of conditions that a SUT must satisfy to allow reliable realization of an AUC. \\
\hline Scenario & $\begin{array}{l}\text { Set of application-driven influencing factors that abstract the AUC for application- } \\
\text { dependent test and evaluation. }\end{array}$ \\
\hline Semi-controlled test environments & Indoor facilities that provide a test area with a modifiable environment configuration. \\
\hline System Specification & Description of the hardware and software configuration of a SUT. \\
\hline System under Test (SUT) & Localization system being tested and evaluated. \\
\hline Testbed & $\begin{array}{l}\text { Combination of test facility, test area, reference system, available ELT and environmental } \\
\text { control parameters. }\end{array}$ \\
\hline Test and evaluation (T\&E) & $\begin{array}{l}\text { Procedure for determining the performance and/or suitability of a localization system } \\
\text { based on empirical experiment data. }\end{array}$ \\
\hline
\end{tabular}

\section{References}

1. Zhang, D.; Xia, F.; Yang, Z.; Yao, L.; Zhao, W. Localization Technologies for Indoor Human Tracking. In Proceedings of the 2010 5th International Conference on Future Information Technology (FutureTech), Busan, Korea (South), 21-24 May 2010, pp. 1-6.

2. Jo, J.; Kim, H.; Yoon, D.; Jang, I. A real-time tracking system of postal motorcyclists. In Proceedings of the 2016 International Conference on Information and Communication Technology Convergence (ICTC), Jeju, Korea (South), 19-21 October 2016, pp. 241-243.

3. Shahroz, M.; Ahmad, F.; Younis, M.S.; Ahmad, N.; Kamel Boulos, M.N.; Vinuesa, R.; Qadir, J. COVID-19 digital contact tracing applications and techniques: A review post initial deployments. J. Transp. Eng. 2021, 5.

4. Mautz, R. Indoor positioning technologies. Habilitation, ETH Zürich, Zürich, 2012.

5. Potortì, F.; Crivello, A.; Barsocchi, P.; Palumbo, F. Evaluation of Indoor Localisation Systems: Comments on the ISO/IEC 18305 Standard. 2018 International Conference on Indoor Positioning and Indoor Navigation (IPIN), Nantes, France, 24-27 September 2018, pp. 1-7.

6. Pasricha, S., Overview of Indoor Navigation Techniques. In Position, Navigation, and Timing Technologies in the 21st Century: Integrated Satellite Navigation, Sensor Systems, and Civil Applications, 1 ed.; Morton, Y.J.; van Diggelen, F.; Spilker, J.J.; Parkinson, B.W.; Lo, S.; Gao, G., Eds.; John Wiley \& Sons Incorporated: Newark, US, 2021; Vol. 2, pp. 1141-1170. 
7. Lymberopoulos, D.; Liu, J.; Yang, X.; Choudhury, R.R.; Handziski, V.; Sen, S. A realistic evaluation and comparison of indoor location technologies. In Proceedings of the 14th International Conference on Information Processing in Sensor Networks (IPSN), Seattle, USA, April 13-16 2015; Nath, S.; Krishnamachari, B.; Rowe, A., Eds.; ACM Press: New York, New York, USA; pp. 178-189.

8. Potortì, F.; Crivello, A.; Palumbo, F. The EvAAL Evaluation Framework and the IPIN Competitions. In Geographical and Fingerprinting Data to Create Systems for Indoor Positioning and Indoor/Outdoor Navigation; Elsevier: Amsterdam, Netherlands, 2019; pp. 209-224.

9. Guo, X.; Ansari, N.; Hu, F.; Shao, Y.; Elikplim, N.R.; Li, L. A Survey on Fusion-Based Indoor Positioning. IEEE Commun. Surv. Tutor. 2020, 22, 566-594.

10. van Haute, T.; de Poorter, E.; Rossey, J.; Moerman, I.; Handziski, V.; Behboodi, A.; Lemic, F.; Wolisz, A.; Wiström, N.; Voigt, T.; et al. The EVARILOS Benchmarking Handbook: Evaluation of RF-based Indoor Localization Solutions. Proceeding of the 2nd International Workshop on Measurement-based Experimental Research, Methodology and Tools (MERMAT), Dubli, Ireland, 7 May 2013.

11. van Haute, T.; de Poorter, E.; Lemic, F.; Handziski, V.; Wirström, N.; Voigt, T.; Wolisz, A.; Moerman, I. Platform for benchmarking of RF-based indoor localization solutions. IEEE Commun. Mag. 2015, 53, 126-133.

12. Potortì, F.; Park, S.; Jiménez Ruiz, A.R.; Barsocchi, P.; Girolami, M.; Crivello, A.; Lee, S.Y.; Lim, J.H.; Torres-Sospedra, J.; Seco, F.; et al. Comparing the Performance of Indoor Localization Systems through the EvAAL Framework. Sensors $2017,17$.

13. Van Haute, T.; De Poorter, E.; Moerman, I.; Lemic, F.; Handziski, V.; Behboodi, A.; Wolisz, A.; Wirström, N.; Voigt, T.; Crombez, P.; et al. EVARILOS Deliverables - D2.4 Final Version of the EVARILOS Benchmarking Handbook, 2015. Available online: https: / / evarilos.eu/deliverables/D2.4_final_version_of_the_handbook.pdf (archived on 02 February 2022).

14. 31, J.S. ISO/IEC 18305:2016-11, International Standard - Information Technology - Real time locating systems - Test and evaluation of localization and tracking systems, 2016.

15. Potorti, F.; Barsocchi, P.; Girolami, M.; Torres-Sospedra, J.; Montoliu, R. Evaluating indoor localization solutions in large environments through competitive benchmarking: The EvAAL-ETRI competition. In Proceedings of the 2015 International Conference on Indoor Positioning and Indoor Navigation (IPIN), Banff, AB, Canada, 13-16 October 2015. IEEE, pp. 1-10.

16. Moayeri, N.; Ergin, M.O.; Lemic, F.; Handziski, V.; Wolisz, A. PerfLoc (Part 1): An extensive data repository for development of smartphone indoor localization apps. In Proceedings of the 2016 IEEE 27th Annual International Symposium on Personal, Indoor, and Mobile Radio Communications (PIMRC), Valencia, Spain, 4-8 September 2019, pp. 1-7.

17. Moayeri, N.; Li, C.; Shi, L. PerfLoc (Part 2): Performance Evaluation of the Smartphone Indoor Localization Apps. In Proceedings of the 2018 International Conference on Indoor Positioning and Indoor Navigation (IPIN), Nantes, France, 24-27 September 2018, pp. $1-8$.

18. Schyga, J.; Hinckeldeyn, J.; Bruss, B.; Bamberger, C.; Kreutzfeldt, J. Application-driven Test and Evaluation Framework for Indoor Localization Systems in Warehouses. In WiP Proceedings of the 2021 International Conference on Indoor Positioning and Indoor Navigation (IPIN), Lloret de Mar, Spain, 29 November-2 December 2021, pp. 1-16. (in press).

19. Raes, W.; Bruycker, J.D.; Stevens, N. A Cellular Approach for Large Scale, Machine Learning Based Visible Light Positioning Solutions. In Proceedings of the 2021 International Conference on Indoor Positioning and Indoor Navigation (IPIN), Lloret de Mar, Spain, 29 November-2 December 2021, pp. 1-6.

20. Van Herbruggen, B.; Fontaine, J.; Poorter, E.D. Anchor Pair Selection for Error Correction in Time Difference of Arrival (TDoA) Ultra Wideband (UWB) Positioning Systems. In Proceedings of the 2021 International Conference on Indoor Positioning and Indoor Navigation (IPIN), Lloret de Mar, Spain, 29 November-2 December 2021, pp. 1-8.

21. Yammine, G.; Alawieh, M.; Ilin, G.; Momani, M.; Elkhouly, M.; Karbownik, P.; Franke, N.; Eberlein, E. Experimental Investigation of 5G Positioning Performance Using a mmWave Measurement Setup. In Proceedings of the 2021 International Conference on Indoor Positioning and Indoor Navigation (IPIN), Lloret de Mar, Spain, 29 November-2 December 2021.

22. Zhou, N.; Lau, L.; Bai, R.; Moore, T. Evolutionary-Inspired Strategy for Particle Distribution Optimization in Auxiliary Particle Filtering Algorithm Based Indoor Positioning. In Proceedings of the 2021 International Conference on Indoor Positioning and Indoor Navigation (IPIN), Lloret de Mar, Spain, 29 November-2 December 2021, pp. 1-8+.

23. Sturm, J.; Engelhard, N.; Endres, F.; Burgard, W.; Cremers, D. A benchmark for the evaluation of RGB-D SLAM systems. In Proceedings of the 2012 IEEE/RSJ International Conference on Intelligent Robots and Systems, Vilamoura-Algarve, Portugal, 7-12 October 2012, pp. 573-580.

24. Bousdar Ahmed, D.; Diez, L.E.; Diaz, E.M.; Garcia Dominguez, J.J. A Survey on Test and Evaluation Methodologies of Pedestrian Localization Systems. IEEE Sens. J. 2020, 20, 479-491.

25. Adler, S.; Schmitt, S.; Wolter, K.; Kyas, M. A survey of experimental evaluation in indoor localization research. In Proceedings of the 2015 International Conference on Indoor Positioning and Indoor Navigation (IPIN), Banff, AB, Canada, 13-16 October 2015, pp. 1-10.

26. Zafari, F.; Gkelias, A.; Leung, K.K. A Survey of Indoor Localization Systems and Technologies. IEEE Commun. Surv. Tutor. 2019, 21, 2568-2599.

27. Mendoza-Silva, G.M.; Torres-Sospedra, J.; Huerta, J. A Meta-Review of Indoor Positioning Systems. Sensors $2019,19$.

28. Mainetti, L.; Patrono, L.; Sergi, I. A survey on indoor positioning systems. In Proceedings of the 2014 22nd International Conference on Software, Telecommunications and Computer Networks (SoftCOM), Split, Croatia, 17-19 September 2014, pp. 111-120. 
29. Zhao, Y.; Smith, J.S.; Karumanchi, S.H.; Vela, P.A. Closed-Loop Benchmarking of Stereo Visual-Inertial SLAM Systems: Understanding the Impact of Drift and Latency on Tracking Accuracy. 2020 IEEE International Conference on Robotics and Automation (ICRA), Paris, France, 31 May-31 August, pp. 1105-1112.

30. Kümmerle, R.; Steder, B.; Dornhege, C.; Ruhnke, M.; Grisetti, G.; Stachniss, C.; Kleiner, A. On measuring the accuracy of SLAM algorithms. Auton. Robot 2009, 27, 387-407.

31. Torres-Sospedra, J.; Silva, I.; Klus, L.; Quezada-Gaibor, D.; Crivello, A.; Barsocchi, P.; Pendão, C.; Lohan, E.S.; Nurmi, J.; Moreira, A. Towards Ubiquitous Indoor Positioning: Comparing Systems across Heterogeneous Datasets. In Proceedings of the 2021 International Conference on Indoor Positioning and Indoor Navigation (IPIN), Lloret de Mar, Spain, 29 November-2 December 2021, pp. 1-8.

32. Mendoza-Silva, G.M.; Richter, P.; Torres-Sospedra, J.; Lohan, E.S.; Huerta, J. Long-Term Wi-Fi fingerprinting dataset and supporting material. Zenodo repository, DOI 10.5281/zenodo.1066041.

33. Liu, Y.; Gao, W.; Hu, Z. A large-scale dataset for indoor visual localization with high-precision ground truth. Int. J. Robot. Res. 2022, 41, 129-135.

34. Potorti, F.; Torres-Sospedra, J.; Quezada-Gaibor, D.; Jiménez, A.R.; Seco, F.; Pérez-Navarro, A.; Ortiz, M.; Zhu, N.; Renaudin, V.; Ichikari, R.; et al. Off-line Evaluation of Indoor Positioning Systems in Different Scenarios: The Experiences from IPIN 2020 Competition. IEEE Sens. J. 2021, pp. 1-1.

35. Pérez-Rubio, M.C.; Losada-Gutiérrez, C.; Espinosa, F.; Macias-Guarasa, J.; Tiemann, J.; Eckermann, F.; Wietfeld, C.; Katkov, M.; Huba, S.; Ureña, J.; et al. A realistic evaluation of indoor robot position tracking systems: The IPIN 2016 competition experience. Measurement 2019, 135, 151-162.

36. Ichikari, R.; Kaji, K.; Shimomura, R.; Kourogi, M.; Okuma, T.; Kurata, T. Off-Site Indoor Localization Competitions Based on Measured Data in a Warehouse. Sensors 2019, 19.

37. Shu, Y.; Xu, Q.; Liu, J.; Choudhury, R.R.; Trigoni, N.; Bahl, V. Indoor Location Competition 2.0 Dataset, 2021. Available online: https:/ / www.microsoft.com/en-us/research/publication/indoor-location-competition-2-0-dataset/ (accessed on 15 February 2022).

38. Andiappan, V.; Wan, Y.K. Distinguishing approach, methodology, method, procedure and technique in process systems engineering. Clean Technol. Environ. Policy 2020, 22, 547-555.

39. Seltzer, M.; Krinsky, D.; Smith, K.; Zhang, X. The case for application-specific benchmarking. In Proceedings of the Seventh Workshop on Hot Topics in Operating Systems, Washington DC, US, 28-30 March, 1999, pp. 102-107.

40. Lemic, F.; Handziski, V.; Wirstrom, N.; van Haute, T.; de Poorter, E.; Voigt, T.; Wolisz, A. Web-based platform for evaluation of RF-based indoor localization algorithms. In Proceedings of the 2015 IEEE International Conference on Communication Workshop (ICCW), London, UK, 8-12 June 2015, pp. 834-840.

41. Filip Lemic.; Vlado Handziski.; Arash Behboodi.; Adam Wolisz.; Tom Van Haute.; Eli De Poorter. EVARILOS Deliverables - D4.3 Report on the Results of the Open Challenge. Available online: https://www2.tkn.tu-berlin.de/tkn-projects/evarilos/index php/deliverables/docs/deliverables.php (archived on 02 February 2022).

42. Filip Lemic.; Vlado Handziski.; Adam Wolisz. EVARILOS Deliverables - D4.3b Report on the Cooperation with EvAAL and Microsoft/IPSN Initiatives, 2015. Available online: https://www2.tkn.tu-berlin.de/tkn-projects/evarilos/index.php/ deliverables/docs/deliverables.php (archived on 02 February 2022).

43. Moayeri, N. Response to "Evaluation of indoor localisation systems: comments on the ISO/IEC 18305 standard". In WiP Proceedings of the 2021 International Conference on Indoor Positioning and Indoor Navigation (IPIN), Lloret de Mar, Spain, 29 November-2 December 2021, pp. 1-6. (in press).

44. de Poorter, E.; van Haute, T.; Laermans, E.; Moerman, I. Benchmarking of Localization Solutions: Guidelines for the Selection of Evaluation Points. Ad Hoc Netw. 2017, 59, 86-96.

45. Reyment, R.A.; Jvreskog, K.G., Aims, Ideas, and Models of Factor Analysis. In Applied factor analysis in the natural sciences; Cambridge University Press: Cambridge, UK, 1993; p. 71-88.

46. El-Haik, B.; El-Haik, B.S. Axiomatic quality: Integrating axiomatic design with six-sigma, reliability, and quality engineering; WileyInterscience: Hoboken, NJ, 2005; pp. 10-15.

47. Sun, L.; Di, H.; Tao, L.; Xu, G. A Robust Approach for Person Localization in Multi-camera Environment. In Proceedings of the 2010 20th International Conference on Pattern Recognition (ICPR), Washington DC, US, 23-26 August 2010, pp. 4036-4039.

48. Umeyama, S. Least-squares estimation of transformation parameters between two point patterns. IEEE Trans. Pattern Anal. Mach. Intell. 1991, 13, 376-380.

49. Lee, C.K.M.; Ip, C.M.; Park, T.; Chung, S.Y. A Bluetooth Location-based Indoor Positioning System for Asset Tracking in Warehouse. In Proceedings of the 2019 IEEE International Conference on Industrial Engineering and Engineering Management (IEEM), Macao, China, 15-18 December 2019, pp. 1408-1412.

50. Reinke, C.; Beinschob, P. Strategies for contour-based self-localization in large-scale modern warehouses. In Proceedings of the 2013 IEEE 9th International Conference on Intelligent Computer Communication and Processing (ICCP), Cluj-Napoca, Romania, 5-7 September 2013, pp. 223-227.

51. Macoir, N.; Bauwens, J.; Jooris, B.; van Herbruggen, B.; Rossey, J.; Hoebekeand, J.; de Poorter, E. UWB Localization with Battery-Powered Wireless Backbone for Drone-Based Inventory Management. Sensors 2019, 19. 
52. Schyga, J. Repository for evaluating indoor localization systems with the T\&E 4iLoc Framework, 2022. GitLab repository: https:/ / collaborating.tuhh.de/w-6/publications/tue4log.

53. Gul, F.; Rahiman, W.; Alhady, S.S.N. A comprehensive study for robot navigation techniques. Cogent Eng. $2019,6$.

54. Khairuddin, A.R.; Talib, M.S.; Haron, H. Review on simultaneous localization and mapping (SLAM). In Proceedings of the 2015 IEEE International Conference on Control System, Computing and Engineering (ICCSCE), Penang, Malaysia, 27-29 November 2015, pp. 85-90.

55. Seow, C.K.; Tan, S.Y. Non-Line-of-Sight Localization in Multipath Environments. IEEE Trans. Mob. Comput. 2008, 7, 647-660.

56. SICK AG. LOCU, Tag-based localization. Available online: https://cdn.sick.com/media/docs/0/40/140/special_information_ locu_en_im0082140.pdf (archived on 26 February 2022).

57. SICK AG. microScan3, Safety laser scanner. Available online: https://www.sick.com/media/docs/7/57/757/Operating instructions_microScan3_Safety_laser_scanner_en_IM0063757.PDF (archived on 26 February 2022).

58. SICK AG. SIM1000, Sensor Integration Machine. Available online: https://cdn.sick.com/media/docs/7/07/307/operating_ instructions_sim1000_fxa_sim1000_fxg_sensor_integration_machine_en_im0083307.pdf (archived on 26 February 2022).

59. SICK AG. LiDAR-LOC, Modular LIDAR localization based on natural contours. Available online: https://cdn.sick.com/media/ familyoverview/2/72/272/familyOverview_LiDAR-LOC_g541272_en.pdf (archived on 26 February 2022).

60. Jia, Y.; Yan, X.; Xu, Y. A Survey of simultaneous localization and mapping for robot. In Proceedings of the 2019 IEEE 4 th Advanced Information Technology, Electronic and Automation Control Conference (IAEAC), Chengdu, China, 20-22 December 2019, 2019, Vol. 1, pp. 857-861.

61. Intel. Intel RealSense Tracking Camera Datasheet. Available online: https://www.intelrealsense.com/wp-content/uploads/20 19/09/Intel_RealSense_Tracking_Camera_Datasheet_Rev004_release.pdf?_ga=2.48506726.1907770152.1645697863-59543418.16 45697863 (archived on 26 February 2022).

62. Chappellet, K.; Caron, G.; Kanehiro, F.; Sakurada, K.; Kheddar, A. Benchmarking Cameras for Open VSLAM Indoors. In Proceedings of the 2020 25th International Conference on Pattern Recognition (ICPR), Milan, Italy, 10-15 Jan. 2021, pp. 4857-4864.

63. IEC/IEEE 61588-2021, IEC/IEEE International Standard - Precision Clock Synchronization Protocol for Networked Measurement and Control Systems, 2021.

64. Quigley, M.; Conley, K.; Gerkey, B.P.; Faust, J.; Foote, T.; Leibs, J.; Wheeler, R.; Ng, A.Y. ROS: an open-source Robot Operating System. ICRA Workshop on Open Source Software, Kobe, Japan ,12-17 May 2009.

65. Hausamann, P.; Sinnott, C.B.; Daumer, M.; MacNeilage, P.R. Evaluation of the Intel RealSense T265 for tracking natural human head motion. Sci. Rep. 2021, 11, 12486.

66. Jordaan, J.P.; Kruger, C.P.; Silva, B.J.; Hancke, G.P. An ultrasonic-based localization system for underground mines. In Proceedings of the 2017 IEEE 15th International Conference on Industrial Informatics (INDIN), Emden, Germany, 24-26 July 2017, pp. 141-146.

67. ALT, H.; GODAU, M. COMPUTING THE FRÉCHET DISTANCE BETWEEN TWO POLYGONAL CURVES. Int. J. Comput. Geom. Appl. 1995, 05, 75-91.

68. Friedrich, J.; Tiemann, J.; Wietfeld, C. Accurate Multi-Zone UWB TDOA Localization utilizing Cascaded Wireless Clock Synchronization. In Proceedings of the 2021 International Conference on Indoor Positioning and Indoor Navigation (IPIN), Lloret de Mar, Spain, 29 November-2 December 2021, pp. 1-8.

69. Mendoza, L.R.; O'Keefe, K. Periodic Extended Kalman Filter to Estimate Rowing Motion Indoors Using a Wearable UltraWideband Ranging Positioning System. In Proceedings of the 2021 International Conference on Indoor Positioning and Indoor Navigation (IPIN), Lloret de Mar, Spain, 29 November-2 December 2021, pp. 1-8.

70. Zhu, N.; Renaudin, V.; Ortiz, M.; Kone, Y.; Ichard, C.; Ricou, S.; Gueit, F. Foot-mounted INS for Resilient Real-time Positioning of Soldiers in Non-collaborative Indoor Surroundings. In Proceedings of the 2021 International Conference on Indoor Positioning and Indoor Navigation (IPIN), Lloret de Mar, Spain, 29 November-2 December 2021, pp. 1-8.

71. Silva, I.; Pendão, C.; Torres-Sospedra, J.; Moreira, A. Quantifying the Degradation of Radio Maps in Wi-Fi Fingerprinting. In Proceedings of the 2021 International Conference on Indoor Positioning and Indoor Navigation (IPIN), Lloret de Mar, Spain, 29 November-2 December 2021, pp. 1-8.

72. Guan, R.; Zhang, A.; Li, M.; Wang, Y. Measuring Uncertainty in Signal Fingerprinting with Gaussian Processes Going Deep. In Proceedings of the 2021 International Conference on Indoor Positioning and Indoor Navigation (IPIN), Lloret de Mar, Spain, 29 November-2 December 2021, pp. 1-8.

73. Kaiser, S.; Wei, Y.; Renaudin, V. Analysis of IMU and GNSS Data Provided by Xiaomi 8 Smartphone. In Proceedings of the 2021 International Conference on Indoor Positioning and Indoor Navigation (IPIN), Lloret de Mar, Spain, 29 November-2 December 2021, pp. 1-8.

74. Raza, A.; Lolic, L.; Akhter, S.; Liut, M. Comparing and Evaluating Indoor Positioning Techniques. In Proceedings of the 2021 International Conference on Indoor Positioning and Indoor Navigation (IPIN), Lloret de Mar, Spain, 29 November-2 December 2021, pp. 1-8.

75. de Wynckel, M.V.; Signer, B. Indoor Positioning Using the OpenHPS Framework. In Proceedings of the 2021 International Conference on Indoor Positioning and Indoor Navigation (IPIN), Lloret de Mar, Spain, 29 November-2 December 2021, pp. 1-8.

76. Shi, L.; Moayeri, N.; Li, C. On the Use of Lookahead to Improve Wi-Fi Fingerprinting Indoor Localization Accuracy. In Proceedings of the 2021 International Conference on Indoor Positioning and Indoor Navigation (IPIN), Lloret de Mar, Spain, 29 November-2 December 2021, pp. 1-8. 
77. Ai, H.; Hu, T.; Xu, T. RAD-GAN: Radio Map Anomaly Detection for Fingerprint Indoor Positioning with GAN. In Proceedings of the 2021 International Conference on Indoor Positioning and Indoor Navigation (IPIN), Lloret de Mar, Spain, 29 November-2 December 2021, pp. 1-8.

78. Yaul, F.M.; Ajamian, T.; Price, M.R.; Palani, S.; Nadeau, P.M.; Yu, T. Multipath-Resilient Unsynchronized 2.4 GHz ISM-Band RF Positioning Using Coherent Echos. In Proceedings of the 2021 International Conference on Indoor Positioning and Indoor Navigation (IPIN), Lloret de Mar, Spain, 29 November-2 December 2021, pp. 1-8.

79. Parralejo, F.; Aranda, F.J.; Paredes, J.A.; Álvarez, F.J.; Morera, J. Comparative Study of Different BLE Fingerprint Reconstruction Techniques. In Proceedings of the 2021 International Conference on Indoor Positioning and Indoor Navigation (IPIN), Lloret de Mar, Spain, 29 November-2 December 2021, pp. 1-8.

80. Aranda, F.J.; Parralejo, F.; Aguilera, T.; Álvarez, F.J.; Torres-Sospedra, J. Finding Optimal BLE Configuration for Indoor Positioning with Consumption Restrictions. In Proceedings of the 2021 International Conference on Indoor Positioning and Indoor Navigation (IPIN), Lloret de Mar, Spain, 29 November-2 December 2021, pp. 1-8.

81. Wei, X.; Wei, Z.; Radu, V. MM-Loc: Cross-sensor Indoor Smartphone Location Tracking using Multimodal Deep Neural Networks. In Proceedings of the 2021 International Conference on Indoor Positioning and Indoor Navigation (IPIN), Lloret de Mar, Spain, 29 November-2 December 2021, pp. 1-8.

82. Bastiaens, S.; Mommerency, J.; Deprez, K.; Joseph, W.; Plets, D. Received Signal Strength Visible Light Positioning-based Precision Drone Landing System. In Proceedings of the 2021 International Conference on Indoor Positioning and Indoor Navigation (IPIN), Lloret de Mar, Spain, 29 November-2 December 2021, pp. 1-8.

83. Almeida, D.; Pedrosa, E.; Curado, F. Magnetic Mapping for Robot Navigation in Indoor Environments. In Proceedings of the 2021 International Conference on Indoor Positioning and Indoor Navigation (IPIN), Lloret de Mar, Spain, 29 November-2 December 2021, pp. 1-8.

84. Xiong, W.; Bordoy, J.; Gabbrielli, A.; Fischer, G.; Schott, D.J.; Höflinger, F.; Wendeberg, J.; Schindelhauer, C.; Rupitsch, S.J. Two Efficient and Easy-to-Use NLOS Mitigation Solutions to Indoor 3-D AOA-Based Localization. In Proceedings of the 2021 International Conference on Indoor Positioning and Indoor Navigation (IPIN), Lloret de Mar, Spain, 29 November-2 December 2021, pp. 1-8.

85. Vey, Q.; Spies, F.; Pestourie, B.; Genon-Catalot, D.; Van Den Bossche, A.; Val, T.; Dalce, R.; Schrive, J. POUCET: A Multi-Technology Indoor Positioning Solution for Firefighters and Soldiers. In Proceedings of the 2021 International Conference on Indoor Positioning and Indoor Navigation (IPIN), Lloret de Mar, Spain, 29 November-2 December 2021, pp. 1-8.

86. Bornholdt, L.; Kaven, S.; Skwarek, V. Adaptive procedure for indoor localization using LoRa devices. In Proceedings of the 2021 International Conference on Indoor Positioning and Indoor Navigation (IPIN), Lloret de Mar, Spain, 29 November-2 December 2021, pp. 1-8.

87. Klipp, K.; Kisand, A.; Wortmann, J.; Radusch, I. Multidimensional In- and Outdoor Pedestrian Tracking using OpenStreetMap Data. In Proceedings of the 2021 International Conference on Indoor Positioning and Indoor Navigation (IPIN), Lloret de Mar, Spain, 29 November-2 December 2021, pp. 1-8.

88. Nakamura, M.; Fujimoto, K.; Murakami, H.; Hashizume, H.; Sugimoto, M. Indoor Localization Method For a Microphone Using a Single Speaker. In Proceedings of the 2021 International Conference on Indoor Positioning and Indoor Navigation (IPIN), Lloret de Mar, Spain, 29 November-2 December 2021, pp. 1-8.

89. Nakamura, M.; Yamasaki, S.; Hashizume, H.; Sugimoto, M. Short-Time and Adaptive Controllable Spot Communication Using COTS Speaker. In Proceedings of the 2021 International Conference on Indoor Positioning and Indoor Navigation (IPIN), Lloret de Mar, Spain, 29 November-2 December 2021, pp. 1-8.

90. Huang, Y.; Shi, D.; Zhang, X.; Aglzim, E.H.; Shi, L. Environment-Aware RSSI Based Positioning Algorithm for Random Angle Interference Cancellation in Visible Light Positioning System. In Proceedings of the 2021 International Conference on Indoor Positioning and Indoor Navigation (IPIN), Lloret de Mar, Spain, 29 November-2 December 2021, pp. 1-8.

91. Tsiamitros, N.; Rumaney, W.; Prehofer, C.; Brunke, J.; Wilz, I.; Pipelidis, G. Evaluation of Non-Intrusive Positioning for Public Environments. In Proceedings of the 2021 International Conference on Indoor Positioning and Indoor Navigation (IPIN), Lloret de Mar, Spain, 29 November-2 December 2021, pp. 1-8.

92. Yao, B.; Li, W.; Wei, D.; Zhang, W.; Li, X. Topology Construction Based on Indoor Crowdsourcing Data using Manifold Learning: Evaluation of Algorithms and Key Parameters. In Proceedings of the 2021 International Conference on Indoor Positioning and Indoor Navigation (IPIN), Lloret de Mar, Spain, 29 November-2 December 2021, pp. 1-8.

93. Abe, K.; Sato, T.; Watanabe, H.; Hashizume, H.; Sugimoto, M. Smartphone Positioning Using an Ambient Light Sensor and Reflected Visible Light. In Proceedings of the 2021 International Conference on Indoor Positioning and Indoor Navigation (IPIN), Lloret de Mar, Spain, 29 November-2 December 2021, pp. 1-8.

94. Josephy, P.; Tanner, S.; Wattenhofer, R. Combined ADS-B and GNSS Indoor Localization. In Proceedings of the 2021 International Conference on Indoor Positioning and Indoor Navigation (IPIN), Lloret de Mar, Spain, 29 November-2 December 2021, pp. 1-8.

95. García-Requejo, A.; Pérez-Rubio, M.C.; Villadangos, J.M.; Gualda, D.; Hernández, A. Positioning Android Devices in Large Indoor Spaces and Transitioning to Outdoors by Sensor Fusion. In Proceedings of the 2021 International Conference on Indoor Positioning and Indoor Navigation (IPIN), Lloret de Mar, Spain, 29 November-2 December 2021, pp. 1-8. 
96. Anagnostopoulos, G.G.; Kalousis, A. ProxyFAUG: Proximity-based Fingerprint Augmentation. In Proceedings of the 2021 International Conference on Indoor Positioning and Indoor Navigation (IPIN), Lloret de Mar, Spain, 29 November-2 December 2021, pp. 1-7.

\section{Short Biography of Authors}

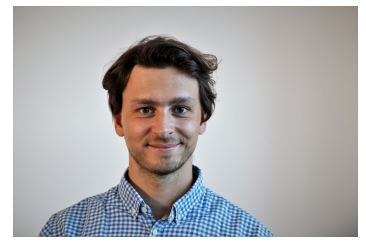

Jakob Schyga, M.Sc. Research Associate at the Institute for Technical Logistics, Hamburg University of Technology. Jakob Schyga studied mechanical engineering and production at the Hamburg University of Technology from 2012 to 2018. Since 2019, Jakob Schyga has been working on the investigation and application of indoor localization systems in warehouses.

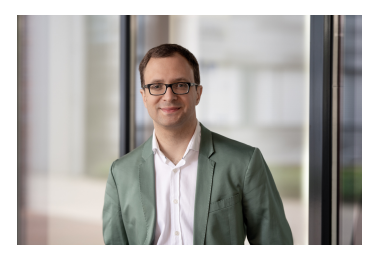

Dr. Johannes Hinckeldeyn Senior Engineer at the Institute for Technical Logistics, Hamburg University of Technology. After completing his doctorate in the UK, Johannes Hinckeldeyn worked as Chief Operating Officer for a manufacturer of measurement and laboratory technology for battery research. Johannes Hinckeldeyn studied industrial engineering, production technology, and management in Hamburg and Münster. Current research focuses in particular on the optimization of logistics processes through digital technologies.

Prof. Dr.-Ing. Jochen Kreutzfeldt Professor and Head of the Institute for Technical Logistics, Hamburg University of Technology. After studying mechanical engineering, Jochen Kreutzfeldt held various management positions at a company group specializing in automotive safety technology. Jochen Kreutzfeldt then took on a professorship for logistics at the Hamburg University of Applied Sciences and became head of the Institute for Product and Production Management. His current research focuses on warehouse and production planning as well as process optimization. 